\title{
Inviscid/Boundary-Layer Aeroheating Approach for Integrated Vehicle Design
}

\author{
Esther Lee* and Kathryn E. Wurster ${ }^{\dagger}$ \\ NASA Langley Research Center, Hampton, VA, 23681
}

\begin{abstract}
A typical entry vehicle design depends on the synthesis of many essential subsystems, including thermal protection system (TPS), structures, payload, avionics, and propulsion, among others. The ability to incorporate aerothermodynamic considerations and TPS design into the early design phase is crucial, as both are closely coupled to the vehicle's aerodynamics, shape and mass. In the preliminary design stage, reasonably accurate results with rapid turn-around times for parametric studies and quickly evolving configurations are necessary to steer design decisions. This investigation considers the use of an unstructured 3D inviscid code in conjunction with an integral boundary-layer method; the former providing the flowfield solution and the latter the surface heating. Sensitivity studies for Mach number, angle of attack, and altitude, examine the feasibility of using this approach to populate a representative entry flight envelope based on a limited set of inviscid solutions. Each inviscid solution is used to generate surface heating over the nearby trajectory space. A subset of a representative entry envelope was explored. Initial results suggest that for Mach numbers ranging from 9-20, a few inviscid solutions could reasonably support surface heating predictions at Mach numbers variation of $+/-2$, altitudes variation of $+/-10$ to $20 \mathrm{kft}$, and angle-of-attack variation of $+/-5^{\circ}$. Agreement with Navier-Stokes solutions was generally found to be within $10-15 \%$ for Mach number and altitude, and $20 \%$ for angle of attack. A smaller angle-of-attack increment than the $5^{\circ}$ considered in this study is recommended. Results of the angle-of-attack sensitivity studies show that smaller increments may be needed for better heating predictions. The approach is well suited for application to conceptual multidisciplinary design and analysis studies where transient aeroheating environments are critical for vehicle TPS and thermal design. Concurrent prediction of aeroheating environments, coupled with the use of unstructured methods, is considered enabling for TPS material selection and design in conceptual studies where vehicle mission, shape, and entry strategies evolve rapidly.
\end{abstract}

\section{Nomenclature}

\begin{tabular}{|c|c|c|c|}
\hline$a_{\infty}$ & speed of sound, $\mathrm{ft} / \mathrm{s}$ & $R e_{\infty}$ & freestream Reynolds number \\
\hline$C_{A}$ & axial force coefficient & $R_{N}$ & nose radius, in \\
\hline$C_{N}$ & normal force coefficient & $s$ & distance from stagnation point along centerline, in \\
\hline$\epsilon$ & emissivity & $T_{\infty}$ & freestream temperature, ${ }^{\circ} \mathrm{R}$ \\
\hline$H$ & altitude, $\mathrm{ft}$ & $V_{\infty}$ & freestream velocity, $\mathrm{ft} / \mathrm{s}$ \\
\hline$l$ & cone length, in & $x$ & body axial distance, in \\
\hline$M_{\infty}$ & freestream Mach number & $\alpha$ & angle of attack, degrees \\
\hline$P_{\infty}$ & freestream pressure, $\mathrm{lbf} / \mathrm{ft}^{2}$ & $\rho_{\infty}$ & freestream density, slugs $/ \mathrm{ft}^{3}$ \\
\hline$q_{w}$ & heat rate at wall, $\mathrm{Btu} / \mathrm{ft}^{2}-\mathrm{s}$ & $\theta_{c}$ & cone half-angle, degrees \\
\hline$q_{s}$ & heat rate at stagnation point, $\mathrm{Btu} / \mathrm{ft}^{2}-\mathrm{s}$ & & \\
\hline
\end{tabular}




\section{Introduction}

A vehicle entering from Low Earth Orbit (LEO) must traverse the entire speed regime from hypersonic to supersonic to transonic, and finally subsonic, as it descends through the atmosphere. Typically, the majority of the laminar convective heating occurs between entry and about Mach 10 of the trajectory. During the hypersonic portion of the flight, the high energy (or enthalpy) of the flow heats the vehicle by convective, and sometimes radiative mechanisms. In most cases, the bulk of the energy contained in the flow is re-radiated, but the remainder is significant at orbital entry velocities and heats up the surface of the vehicle. Therefore, it is critical for the vehicle designer to account for this in an integration of vehicle shape, entry profile, and thermal protection or control. The Thermal Protection System (TPS) prevents the harsh entry heating environment from damaging the vehicle, in particular the structure, avionics, payload, and personnel. An understanding of aerothermodynamics is key to the integrated vehicle system design.

Aerothermodynamics combines the thermodynamics and aerodynamics disciplines; it is most often applicable in hypersonic flight where high temperature gas and boundary-layer effects are present. These effects strongly influence the flow and must be captured accurately for better understanding prior to and during the vehicle design process. Experimental testing and computational numerical simulations are options to study fluid flow. However, because flight tests and wind-tunnel facilities are costly and cannot typically simulate the high-enthalpy and real-gas environments of flight at Earth entry velocities, it is necessary to rely on computational tools to predict the flow and external heating environment accurately. Although Navier-Stokes Computational Fluid Dynamics (CFD) codes or Viscous Shock Layer codes yield high-fidelity solutions, the computational resources and time required for each accurate solution are currently prohibitive for use during the conceptual design phase.

While accurate predictions of the flow behavior are important throughout the vehicle design process, engineering codes employing approximate methods with appropriate assumptions can be used to perform calculations at the conceptual and even preliminary design stage, limiting computational costs and resource requirements; high-fidelity CFD tools are currently best suited for use in the later design stages. The resources required to populate evolving entry flight envelopes with the sufficient number of CFD solutions for TPS/thermal design are prohibitive at this time. Engineering codes can provide reasonably accurate solutions sufficient for parametric studies and to guide each iteration of a rapidly changing design in a relatively short amount of time. However, it is important to note that engineering codes do not include all the equations and terms describing the physics; instead they are generally approximations based on certain assumptions and are often empirically derived. It is important for the user to become aware of these assumptions to ensure the engineering method application is sensible, realistic, and within its range of applicability.

\section{Background}

In the 1990s, the Reusable Launch Vehicle Technology Program was initiated by NASA and industry partners to replace the Space Transportation System (Space Shuttle orbiter fleet). ${ }^{1}$ Advanced conceptual vehicles, such as the $\mathrm{X}-33^{2}$ and $\mathrm{X}-34,{ }^{3}$ were part of the program intended to provide cost-effective, yet reliable access to space. These design efforts shed light on an important requirement for development and application of computational aerothermodynamic tools in a multidisciplinary design environment: the need to respond to evolving designs by providing accurate and transient aeroheating environments within a tight time-frame ${ }^{2}$ and at the same time optimizing as part of a system and interacting with other design disciplines. Typically, computational solutions represent point solutions on a given geometry at a given flight condition and vehicle attitude. TPS design requires environments along a flight profile in order to capture

both peak temperatures and aeroheating loads, informing TPS material selection and enabling analysis of the material response (including structural temperatures). The requirement for transient environments led to and encouraged different aerothermal engineering tool approaches, ${ }^{3-5}$ a mix of approximate methods, Navier-Stokes/CFD codes and experimental data (where available), to be used at the conceptual design stage to analyze and design TPS and interact with other disciplines. For cases in which the vehicle mission allows a vehicle of sufficient aerodynamic performance, the three disciplines - aerothermodynamics, TPS design, and trajectory control - can be integrated to provide an optimal TPS/entry strategy thus reducing TPS weight requirements. ${ }^{6}$ More recent efforts ${ }^{7,8}$ to integrate aerothermodynamics and TPS sizing for multidisciplinary design and analysis optimization (MDAO) purposes also emphasized the importance of the 
system-of-systems analysis environment for conceptual design. The approach to obtaining surface heating environments as discussed in this paper is intended for implementation in a multidisciplinary analysis tool to support conceptual vehicle design, specifically TPS and trajectory design strategies.

Validated against experimental data and computational solutions, engineering codes have been demonstrated as good alternatives to offer reasonable fidelity answers for conceptual designs. ${ }^{9-11}$ These codes use approximate techniques to estimate convective heating over portions of the vehicle that can be modeled by simple analytical models: nose, wing leading edges, body acreage, etc. Stagnation point, ${ }^{12,13}$ laminar ${ }^{14}$ and turbulent flow, as well as flow characteristics, such as variable entropy effects on boundary-layer edge conditions ${ }^{15}$ can be captured. These methods provide a simple approach to solutions based on a set of assumptions, which must be known to the aerothermodynamic discipline expert in the loop. In the inviscid/boundary-layer approach, the crossflow velocity is negligible and therefore the 3D boundary-layer equations can be simplified to axisymmetric flow boundary-layer equations, ${ }^{16}$ limiting the side-slip angle to zero. Boundary-layer edge properties are determined by an inviscid flowfield solution. The inviscid/boundary layer approach sometimes incorporates an approximate procedure that accounts for variable-entropy effects in the viscous flow for convective heating on blunt bodies. ${ }^{15,16}$ Previous work applied the inviscid/boundarylayer approach to structured grids ${ }^{17,18}$ on vehicles such as the X-33 ${ }^{2}$ and X-34. ${ }^{19}$ More recent efforts have extended this approach to incorporate the integration with unstructured inviscid flowfield solutions derived from unstructured surface meshes. ${ }^{20}$ This paper will discuss the inviscid/boundary layer approach applied for unstructured surface meshes. It should be noted that the variable entropy feature is not currently implemented in the unstructured code used here, and therefore not considered in the present study.

The unstructured approach offers inherent advantages over the structured single-block approach for applications where analysis of complex and rapidly evolving designs is critical; however, calculating heating solutions with unstructured (purely tetrahedral) grids has been proven to be very challenging as the skewness and irregular nature yield large errors in surface pressure and heat-transfer rates. ${ }^{21,22}$ Moreover, the high aspect ratios of unstructured tetrahedral flowfield grids introduce significant inviscid flux errors across shocks, making them undesirable for aerothermal computational purposes. However, the post-shock error seems to be much smaller and more controllable for hexahedral grids. ${ }^{23}$

Four inviscid solvers were initially considered for the generation of the inviscid solutions required to obtain the boundary-layer edge conditions: CART3D, ${ }^{24}$ FELISA, ${ }^{25}, 26$ FUN3D, ${ }^{27}$ and LAURA (Langley Aerothermodynamic Upwind Relaxation Algorithm). ${ }^{28,29}$ Three were removed from consideration: FELISA because it is no longer supported, FUN3D, because the inviscid mode has not been verified, and finally LAURA. LAURA's inviscid mode uses triangulation to convert to unstructured grid format, in which case there would be no clear advantage over the direct implementation of the high-fidelity, viscous LAURA solution capability. CART3D ${ }^{24}$ was selected for application in this study. Key considerations for its selection were CART3D's basis in an unstructured Cartesian grid, rapid generation and adaptability to complex shapes, together with the existence of an active support community. ${ }^{24}$ These characteristics are enabling for application in conceptual design studies where configurations and missions evolve rapidly.

\section{Objective}

Implementation of a mid-fidelity approach to provide transient aeroheating environments to support TPS design for entry vehicles in the conceptual design phase is desired to facilitate MDAO for complex advanced vehicles. The objective here is to develop the necessary insight required to begin the implementation of an automated process directed towards generating reasonable (mid-fidelity) aeroheating results within a multidisciplinary analysis (MDA) environment, supporting TPS design for iterative conceptual design (using unstructured surface grids). This improvement in fidelity will enable faster, more reliable results compared to lower-fidelity engineering methods. The desired outcome, and ultimate goal of implementing the mid-fidelity tool in the multidisciplinary analysis environment, is to populate an aerothermal database for analysis and integration with TPS tools.

\section{A. Approach}

The learning process will be briefly described as insight into the future formulation of an automated process to generate heating solutions is gained. A simple sphere-cone geometry is used to demonstrate the intricate steps required and the care that must be exercised to successfully obtain aeroheating solutions using the 
proposed mid-fidelity approach. The aerothermodynamic analysis for this conceptual design approach begins with an unstructured surface mesh that is subsequently passed to an inviscid CFD code to generate flowfield solutions, which in turn provide the boundary conditions for calculation of heating solutions. Interpolation procedures are used to fill in the design space between the computed heating solutions chosen to populate the expected flight envelope. The aeroheating environments thus generated are intended for integration with existing TPS design, analysis, and sizing tools. The presence of an engineer-in-the-loop to perform sanity checks and to evaluate results throughout the automated process is an acknowledged design requirement and is essential to ensure realistic and accurate aeroheating environments for TPS analysis and design. Accuracy and limits to the approach are also investigated. Lastly, results are verified using a high-fidelity benchmark code and are also validated against wind-tunnel data.

\section{B. Assumptions}

In order to use this mid-fidelity tool to obtain the aeroheating solution, a few assumptions are made. The flow is assumed to be laminar for the purposes of this study and initial demonstration of the approach. Studies have shown that, for vehicles with reasonable aerodynamic performance (winged or lifting bodies), entry trajectories can be tailored to delay transition such that the TPS design is dominated by the laminar portion of the flight profile. ${ }^{30,31}$ To simplify the problem, a no-sideslip condition is assumed and all surfaces are considered to be non-catalytic. High-temperature/energy flows are associated with the increased likelihood of chemical-physical interaction of the molecules in the flow. The gas (air in this case) becomes chemically reactive at the high temperatures associated with entry from LEO. Here, dissociation reactions are assumed to have settled into equilibrium flow. In hypersonic flow, the shock wave lies very close to the body and, when high temperature and equilibrium effects are considered, the shock layer becomes very thin. The thin shock layer complicates the physics of the problem as the shock wave and the viscous boundary-layer growing on the body surface merge, also known as entropy layer swallowing. ${ }^{32}$ This phenomenon has significant effect on heating-rate calculations. However, this study does not include the effects of entropy layer swallowing as the techniques to do such calculations on unstructured grids are not readily available. ${ }^{20}$ This study is exclusively directed towards the design of TPS for LEO entry vehicles (entry velocity: approximately $26 \mathrm{kft} / \mathrm{s}$ ). Direct Simulation Monte Carlo methods are not considered as vehicle TPS is generally driven by environments in the continuum flow regime. In addition, radiative heating is generally insignificant compared to convective heating at LEO entry conditions. Radiative heating is thus ignored for the purpose of this study.

\section{Tools Introduction}

This section introduces the tools and methods selected for use in this study. While there are certainly better and more diverse choices/capabilities with commercial off-the-shelf software/tools for the surface mesh generator, this feasibility study is limited to tools readily available within the environment in which the MDA process of interest is currently implemented, and those that can be obtained at no additional cost.

IDEA $^{7}$ (Integrated Design \& Engineering Analysis) is a multidisciplinary analysis tool implemented in the Adaptive Modeling Language (AML) ${ }^{33}$ framework. IDEA integrates many subsystem analysis tools into one design environment, capable of creating a vehicle model and calling different analysis tools to be used within one interface. Geometry, packaging, propulsion, trajectory, aerodynamics, aerothermodynamics, engine and airframe subsystem design, thermal and structural analysis, and vehicle closure capabilities are incorporated in a way such that data is shared seamlessly across the design disciplines.

CART3D, a high-fidelity inviscid code, developed at the NASA Ames Research Center, uses Euler equations for flow solving. The code has been applied extensively in the subsonic and transonic regime for aerodynamic analysis; in recent years, its application has extended to the hypersonic regime (using perfect gas or effective gamma). CART3D takes a triangulated, watertight surface mesh (i.e. the body exists as one piece with no gaps through which a fluid could "leak") and creates an unstructured Cartesian volume grid for flow calculations. CART3D has two grid adaptations to automatically build the volume grid: 1) adjoint-based adaptation to minimize errors for target aerodynamic coefficients and 2) feature-based adaptation (in development) to refine the flowfield grid to flow features, such as shocks and expansions. An equilibrium-air-chemistry flow solver package is also available to account for the air chemistry within the shock layer in high-speed, high-temperature flow.

LAURA (Langley Aerothermodynamic Upwind Relaxation Algorithm) is a well-validated industry-standard Navier-Stokes code that has been used extensively for both Earth and planetary entry vehicles including the 
Shuttle orbiter, ${ }^{34}$ Crew Exploration Vehicle, ${ }^{35}$ and Mars Science Laboratory, ${ }^{36}$ to name a few. The code relies on structured grids, can execute in viscous or inviscid mode, and includes fully-catalytic and finite-rate chemistry. It contains perfect gas, equilibrium, thermal and chemical nonequilibrium air models, as well as gas models for other major planets. The code can perform grid adaptation during solution convergence to resolve boundary-layer and bow-shock gradients.

UNLATCH $^{20}$ (UNstructured Langley Approximate Three-dimensional Convective Heating) is an integral boundary-layer code developed at NASA Langley Research Center to obtain aeroheating solutions over an unstructured surface grid using an inviscid flowfield solution. This code allows for rapid computation of heating over generalized 3D vehicles at angle of attack and is based on the axisymmetric analog for a 3D boundary layer, which neglects viscous crossflow in the boundary layer. As stated in the assumptions for this investigation, entropy layer swallowing is currently not included. The code locates the stagnation point and calculates the region around the stagnation point, then calculates the heating along the inviscid surface streamlines along the body. Because UNLATCH is very sensitive to the small velocities in the stagnation area, it is critical to have a well-resolved inviscid solution, especially near the stagnation region. The accuracy of UNLATCH is typically $10-20 \%^{\text {a }}$ with respect to LAURA. An earlier structured grid version of this code is called $\mathrm{LATCH}^{17}$ (Langley Approximate Three-dimensional Convective Heating). LATCH has been used for vehicle design studies such as the $\mathrm{X}-34,{ }^{3}$ where the Outer Mold Line (OML) is fixed, but the structured methods do not lend themselves to rapidly evolving vehicle designs where significant changes in the OML may occur.

\section{Inviscid-Flow/Boundary-Layer Approach}

This section describes in detail the iterative process required to generate an acceptable aeroheating solution. First, the generic process to obtain an aeroheating solution is discussed: an inviscid solution is generated through CART3D and provided to UNLATCH as boundary-layer edge conditions for heating calculations. Guidelines to obtaining quality inviscid solution and, in turn quality heating solutions, are presented. The evaluation processes used to determine "acceptable" inviscid and heating solutions are discussed, including remedies employed to improve those solutions. A 15-degree sphere-cone with nose radius of 1 inch and length of 30 inches is used to aid the process demonstration and discussion.

\section{A. Process Overview}

The procedure developed to obtain heating on a vehicle surface using the inviscid/boundary-layer method is illustrated by the flow diagram shown in Fig. 1. First, a single, watertight unstructured 3D surface mesh of the sphere-cone is interactively created in IDEA, where the user can input the desired limits of surface triangle sizes. The surface mesh shown in Fig. 2 was created using the meshing utility that is included in the AML package. That utility does not incorporate a mesh growth capability to allow smooth transition in cell size. Thus, the abrupt change in cell size near the sphere cone juncture. Although the high aspect-ratio triangles at the discontinuity in cell size could undermine the heating solutions, this was found not to be an issue for the demonstration case used here.

CART3D creates a volume grid based on the surface mesh provided, and computes the inviscid flowfield solution on that grid. UNLATCH calculates the heating along streamlines, starting in the stagnation area. Therefore, it is absolutely critical that the flow in the stagnation region be well-resolved in the inviscid solution. Because the velocities in the stagnation area are small, even very slight inaccuracies can have significant impact on the accuracy of the overall heating solution.

The resolution around the stagnation region and within the shock layer must be well defined. Expert ${ }^{\mathrm{b}}$ opinion suggests a minimum resolution of approximately 10-20 volume cells in the shock layer, similar to the example in Fig. 3(a). Additionally, it is suggested that in the stagnation area, the size ratio between the surface triangles and volume cells must be at least 1:1, as depicted in Fig. 3(b). Two to three surface triangles per volume cell is preferred ${ }^{\mathrm{b}}$. For larger curvature, an even higher ratio of triangles-to-cell is recommended ${ }^{\mathrm{b}}$. Assessment of the initial volume grid can provide insight into the approximate surface triangle and volume cell size required to satisfy these criteria for obtaining a reasonably accurate aeroheating solution. Based on

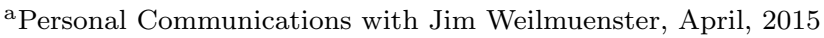

bPersonal Communications with Jim Weilmuenster, February 2015
} 


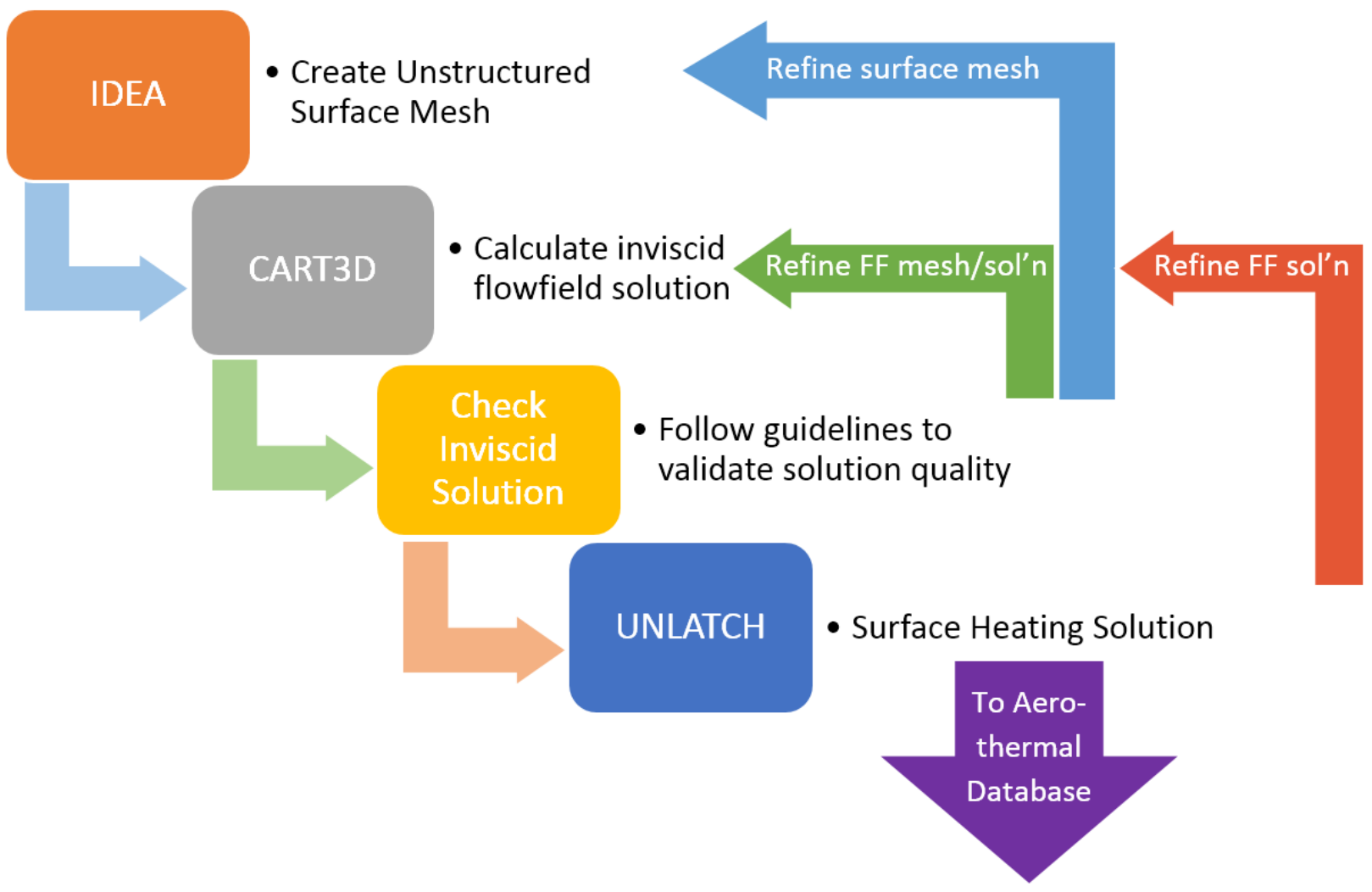

Figure 1: Inviscid-Flow/Boundary-Layer Process Flow Chart

these guidelines, it can be concluded that the surface mesh resolution in the stagnation region is driven by the volume cell size and the shock-standoff distance.

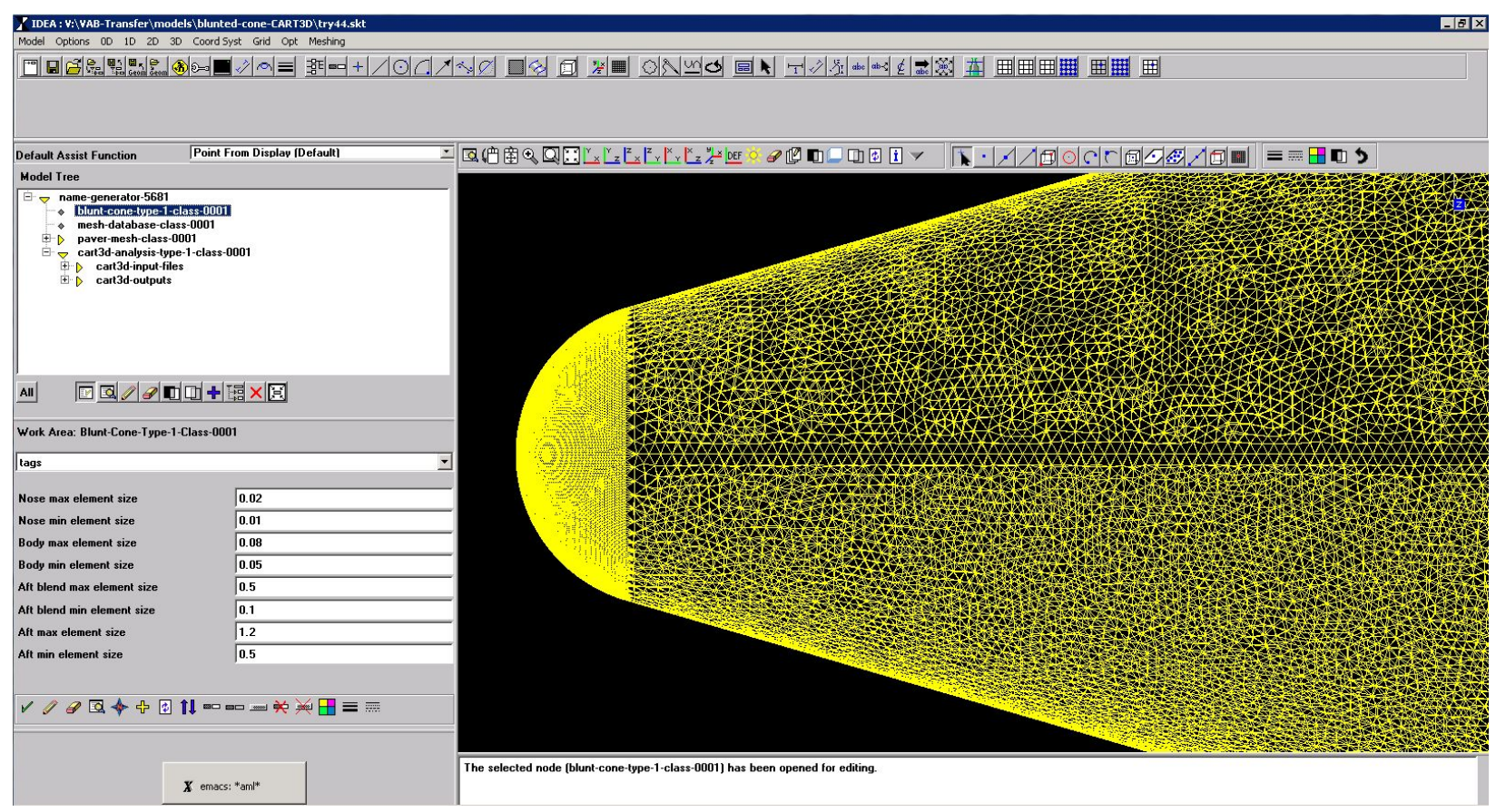

Figure 2: IDEA: multidisciplinary analysis tool interface showing sphere-cone surface mesh 


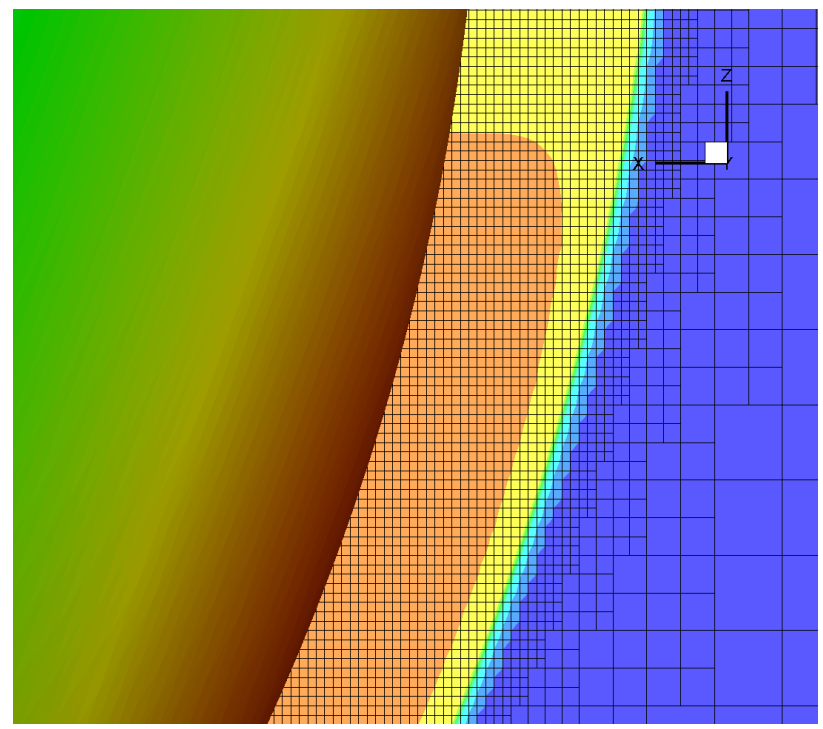

(a) Ideal volume cell count in shock layer

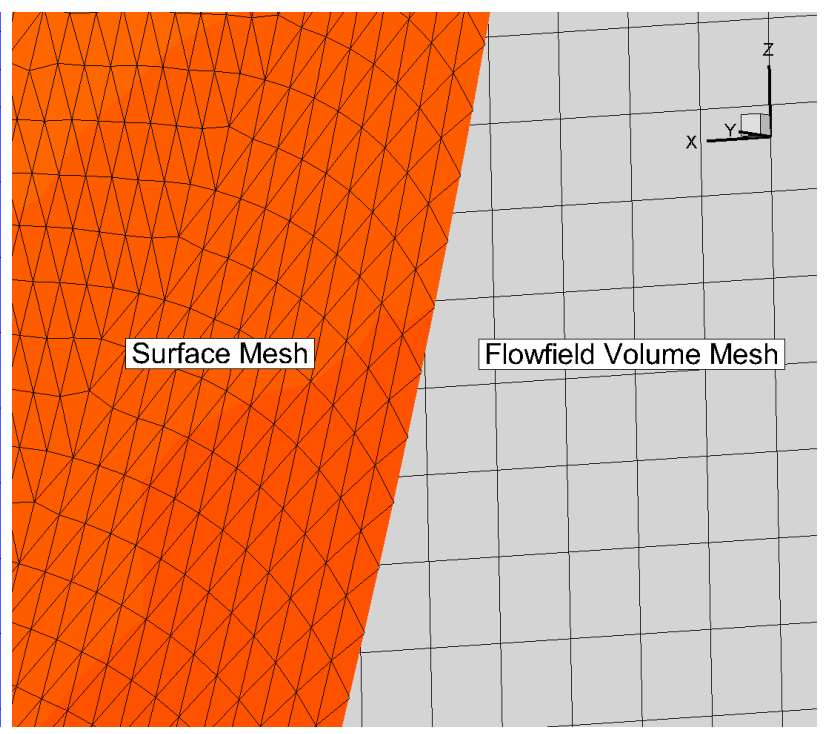

(b) Ideal size ratio between volume cells and surface triangles

Figure 3: Guidelines for volume cells and surface mesh interface

After CART3D computes the inviscid solution from the initial volume grid with the user's input of Mach Number, angle of attack, and specific heat ratio, a qualitative check is performed to determine the preliminary acceptability of the inviscid solution. If the inviscid solution quality is questionable (i.e. does not meet the criteria for a "good" inviscid solution as noted previously), then either the surface or flowfield mesh requires refinement and/or the inviscid flowfield solution requires better resolution. Several features within CART3D allow some form of user control of the volume grid generation to refine the volume grid. One of the features includes defining refinement volume boxes that force CART3D to create more cells within the bounded volume. Another grid-control option is to use either the adjoint-based or the feature-based grid refinement routine. The adjoint-based routine refines the initial mesh to a specific output functional; force and moment coefficients are a few of the functionals that CART3D considers. This adaptation feature takes the initial flowfield mesh and refines the grid such that the discretization errors in the flow solution are reduced, achieving a more accurate solution. In the adjoint adaptation, the grid is refined, solved, and refined again for a user-specified number of iterations.

Another option for grid adaptation is the feature-based routine, which refines to flow features such as shocks and expansions. Similar to the adjoint-based routine, the grid is solved, refined, and solved again for a specified number of iterations. The equilibrium-air-chemistry flow solver (beta version) is used here for the feature-based adaptation rather than the built-in flow solver that uses an effective gamma to perform the inviscid solution calculations. The equilibrium-air-chemistry flow solver accounts for high-temperature gas effects. At higher Mach numbers, dissociation may occur in the high-temperature gas. Depending upon the configuration, the nonequilibrium effects on heating may be present over a good portion of the vehicle. The equilibrium-air-chemistry assumption, implicit to this study, is appropriate for the conceptual design phase as it generally yields a conservative solution. This iterative process to improve the quality of the inviscid solution continues until the solution quality is acceptable.

Once the inviscid solution is deemed to be of acceptable quality, it is transformed for input to the aeroheating tool, UNLATCH, where surface heating solutions are generated. The flowfield solution is converted into FELISA format using a conversion utility code. The utility code, written in Fortran, transforms the CART3D coordinate system into that of UNLATCH. In addition, the utility code trims the inviscid solution at the symmetry plane into a half body solution, and cuts at user-specified lengths in the axial and normal directions. Freestream conditions are input, a wall-temperature boundary condition is chosen and the air chemistry option (perfect gas or equilibrium) is selected. UNLATCH outputs include the surface heating solution, the boundary-layer solution, and the heating along a symmetry plane cut on the surface. If the resulting surface heating solution is not deemed to be acceptable, additional iterations are performed to produce an inviscid solution with better quality. 


\section{B. Steps to Ensure Inviscid Solution Quality}

Although a CART3D solution may appear to be acceptable qualitatively, the derived UNLATCH solution may not necessarily yield a "good" answer. A 15-degree sphere-cone with nose radius of 1 inch and length of 30 inches is used to aid the process demonstration and discussion. The freestream flight condition for the demonstration case is summarized in Table 1.

Table 1: Freestream Flight Condition for 15-degree Sphere-cone Demonstration Case ${ }^{20}$

\begin{tabular}{ccccc}
\hline \hline $\begin{array}{c}\text { Altitude } \\
f t\end{array}$ & $\begin{array}{c}\text { Temperature } \\
{ }^{\circ} R\end{array}$ & $\begin{array}{c}\text { Pressure } \\
\text { lbf/ft }\end{array}$ & Mach & $\begin{array}{c}\alpha \\
\text { degrees }\end{array}$ \\
\hline $180000 \mathrm{ft}$ & 470.062 & 0.903836 & 13.16 & 20 \\
\hline
\end{tabular}

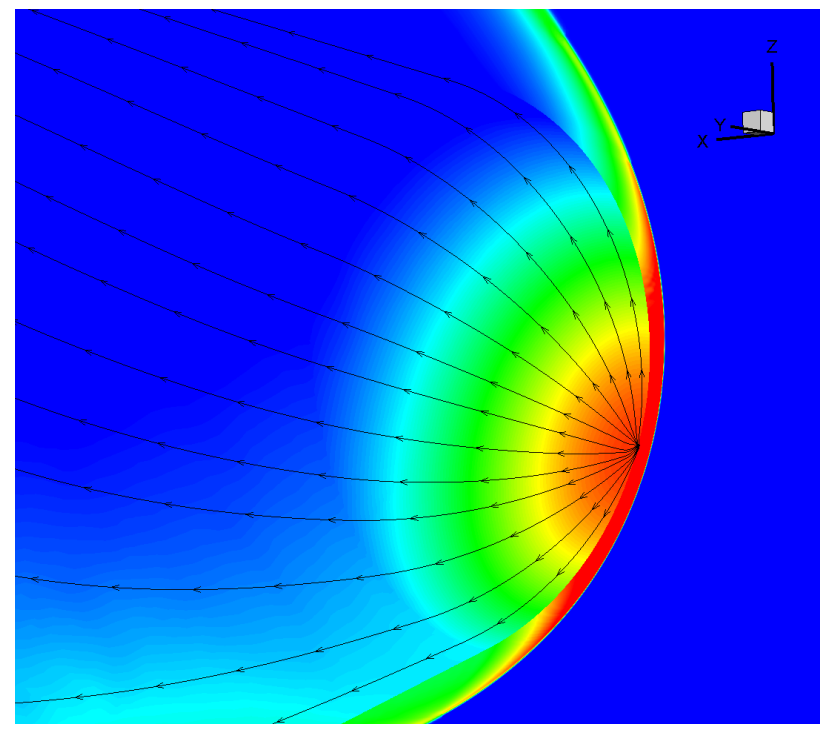

(a) Accepable CART3D Solution

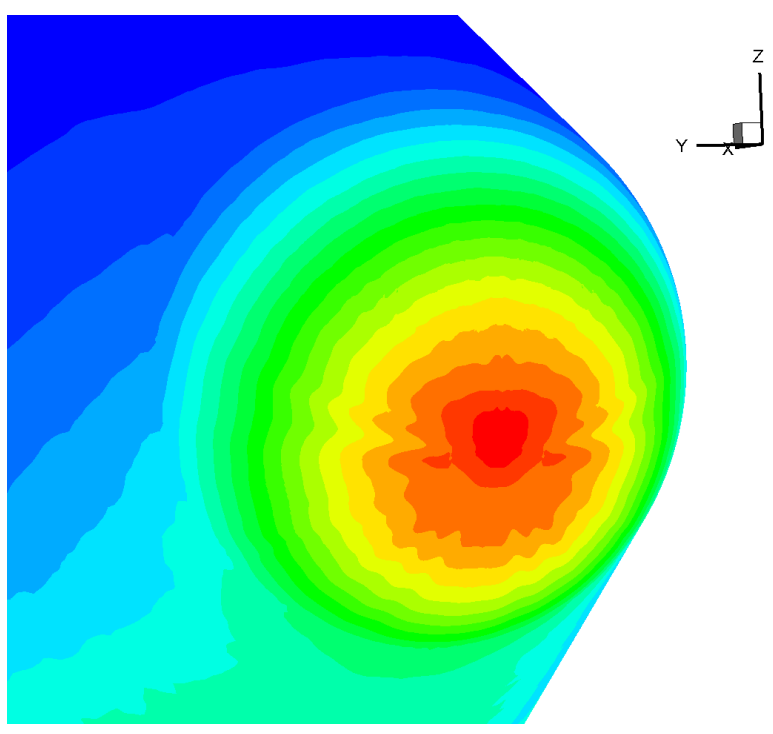

(b) Bad UNLATCH Output

Figure 4: A well-behaved inviscid solution does not guarantee a good surface heating solution

Figures 4(a) and 5(a) shows pressure on the sphere-cone surface and density in the flowfield for the demonstration case. In both cases the pressure distributions and streamlines appear to be well-behaved. However, the "flower-like" pattern in the stagnation heating solution in Fig. 4(b) is clearly physically unrealistic. When an UNLATCH heating solution appears physically unrealistic like that shown in Fig. 4(b), the inviscid flowfield mesh setup must be reassessed and additional effort must be applied to refine the inviscid solution. Whenever an unacceptable UNLATCH solution results, potential remedies lie in improvements in the inviscid solution. Remedies include: checking the volume mesh setup to ensure the volume cells are indeed cubes, expanding the refinement boxes in the stagnation area to make sure the flow in the stagnation region is well-captured, adding extra flow solver iterations to obtain a more converged solution, and adding extra adaptation cycles so that CART3D refines the flowfield grid. In addition to the examination of the flowfield in a qualitative sense, the pressure distribution, particularly along the centerline, can also provide insight to determine whether the solution is sufficiently well-resolved. An unsatisfactory pressure distribution will almost universally lead to a poor outcome from the associated UNLATCH analysis. The iterative process of obtaining and checking the quality of the inviscid solution, and obtaining UNLATCH solutions continues until UNLATCH yields a realistic heating solution. Procedures such as those described here were employed, leading to the results shown in Fig. 5 for the demonstration case illustrated in Fig. 4. The refined solution (Fig. 5(b)) shows significant improvement, yielding a well-behaved and physically realistic heating distribution over the surface. The heating solution is symmetric and smooth, a result expected for axisymmetric flow over a sphere cone. 


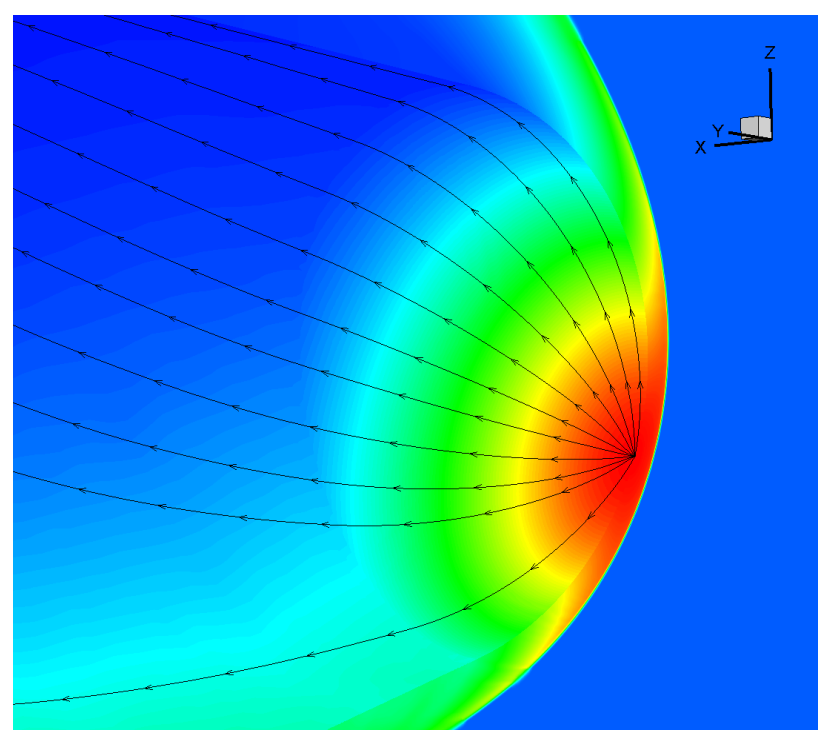

(a) Accepable CART3D Solution

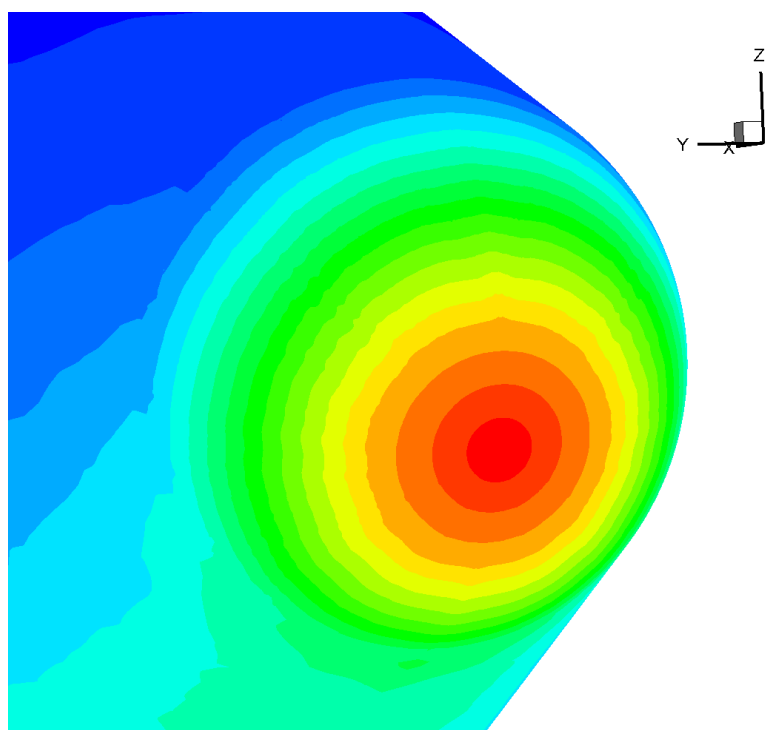

(b) Good UNLATCH Output

Figure 5: Realization of a good solution occurs only when an acceptable UNLATCH solution is observed

For a simple sphere-cone geometry with a 14-million-cell volume mesh, CART3D takes 2 hours to converge to a well-resolved equilibrium inviscid solution on an 80-processor Linux machine, while UNLATCH takes about 3-5 minutes to calculate a heating solution running on a single processor. For this demonstration case, LAURA, using 8 processors, generates a solution in approximately 5 hours. It should be noted that these times cannot be compared directly. In order to populate a flight envelope, a full LAURA solution would be required at each design condition. The inviscid/boundary layer approach would also require an UNLATCH solution at every condition, but multiple UNLATCH cases can be derived from a single CART3D solution. The computational time for UNLATCH is negligible compared to either the LAURA solution or the inviscid CART3D solution. Additional computational resources could significantly improve turn-around time in the conceptual design stage should the proposed approach be implemented in an MDA or MDAO design environment.

\section{Verification and Validation of UNLATCH Heating Solutions}

\section{A. Navier-Stokes Solutions Verification}

LAURA solutions were obtained and used as benchmark validation cases for comparison with the inviscid/boundary layer solutions for the Mach 13.16 demonstration case shown in Table 1. The LAURA volume grid dimension was 60 cells along the cone length, 30 cells circumferentially, and 60 cells into the flow. The verification cases are modeled with nonequilibrium air and a fully-catalytic wall boundary condition in laminar flow. This approach was chosen as it most closely models the equilibrium prediction, which is no longer explicitly available in the most recent version of LAURA.

The stagnation and recompression areas were difficult to capture due to the constant entropy limitation in UNLATCH. Noise in the UNLATCH surface heating along the centerline, shown in Fig. 6(a), is expected as the unstructured grid approach combined with the mesh-growth limitation for the surface mesh likely contributes to difficulties in capturing flow features. If the signature of recompression along the centerline differs significantly from that in the LAURA solution, it is likely an indication that the inviscid solution is not sufficiently resolved, particularly with respect to pressure. In this situation, a quick look at the CART3D pressure plot behavior (Fig. 6(b)) can provide sufficient insight to determine whether the flowfield solution needs to be more resolved. Another iteration of adaptation to "smooth" out the volume cell sizes within the shock layer generally reduces the noise in the pressure plot.

Figure 7 compares the UNLATCH prediction for the windward centerline heating to that of the benchmark LAURA solution for the Mach 13.16, $\alpha=20^{\circ}$ demonstration case. Excellent agreement is observed between 


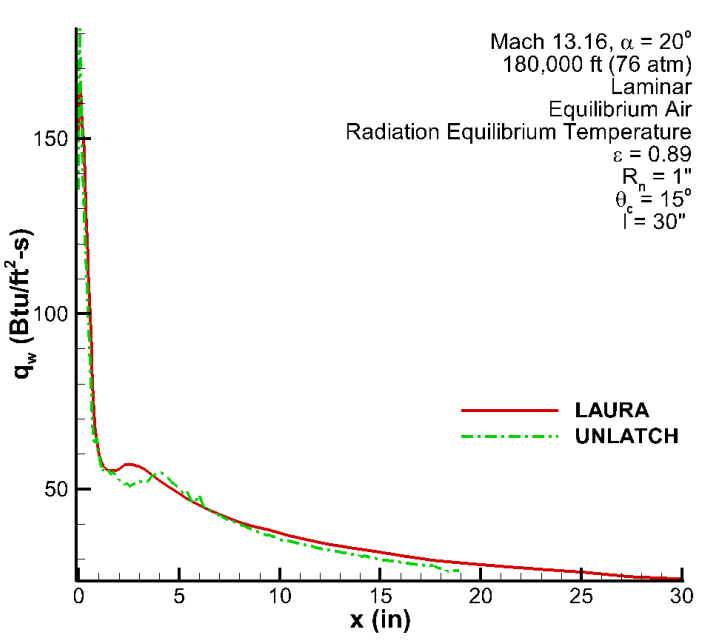

(a) LAURA and UNLATCH comparisons

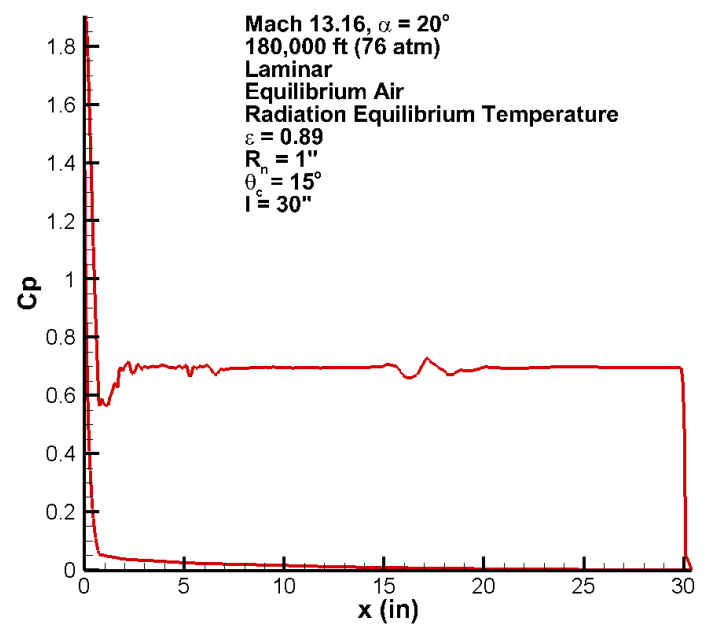

(b) Pressure plot from CART3D inviscid solution

Figure 6: Using pressure distribution to verify inviscid solution quality

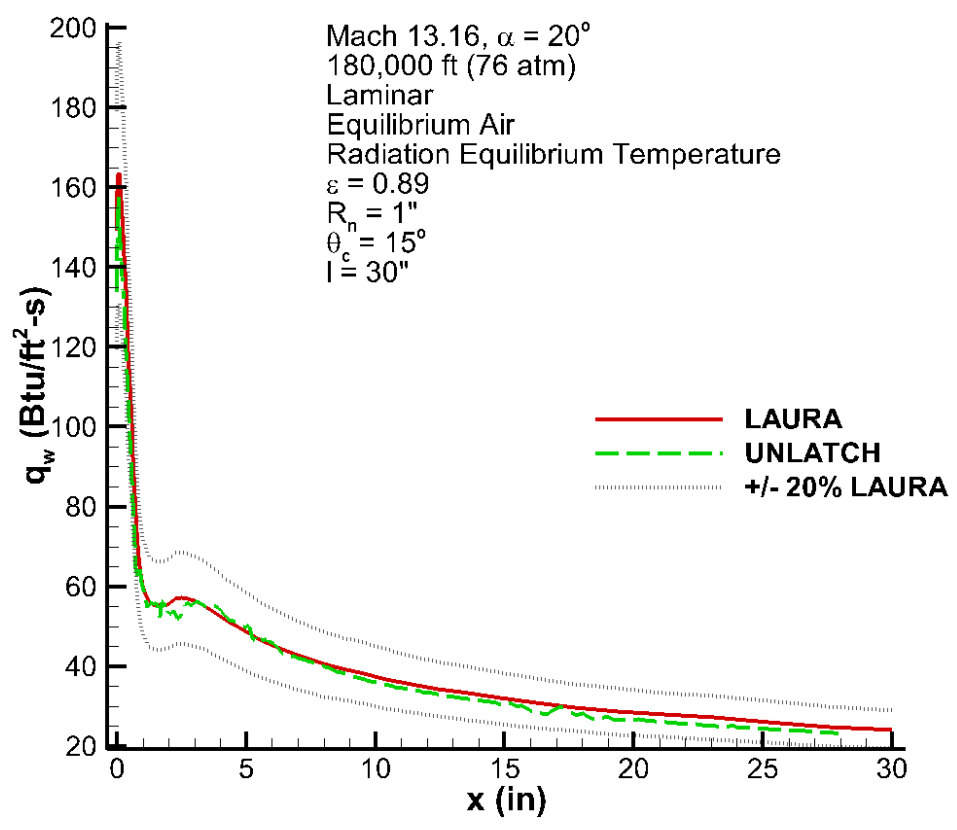

Figure 7: Benchmark comparison along windward centerline for Mach 13.16 case at 180,000 $\mathrm{ft}, \alpha=20^{\circ}$

the UNLATCH prediction and LAURA solution. Along the windward centerline, the peak in heat rate at $x$ $=0.508$ inches indicates the location of the stagnation point. The dotted lines represent a $20 \%$ uncertainty band on the LAURA solution, within which acceptable UNLATCH results are expected to fall. As expected, the heat rate decreases as the flow expands around the nose, and increases slightly again when the flow reaches the recompression point on the lower surface. As the flow travels downstream along the body, it converges to a sharp-cone constant-entropy condition. 
B. Wind-Tunnel Data Validation

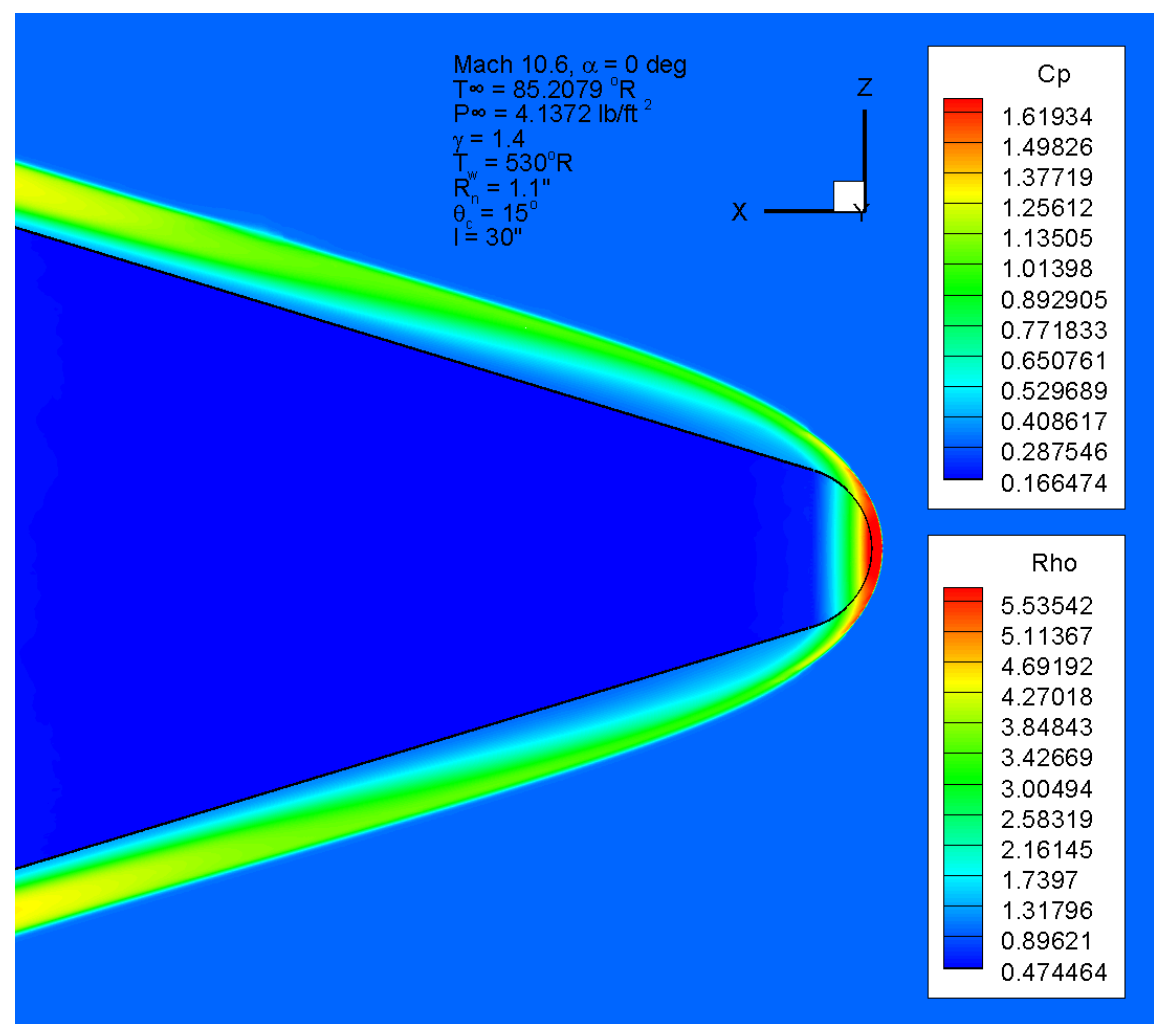

Figure 8: Inviscid Solution for Wind-Tunnel case
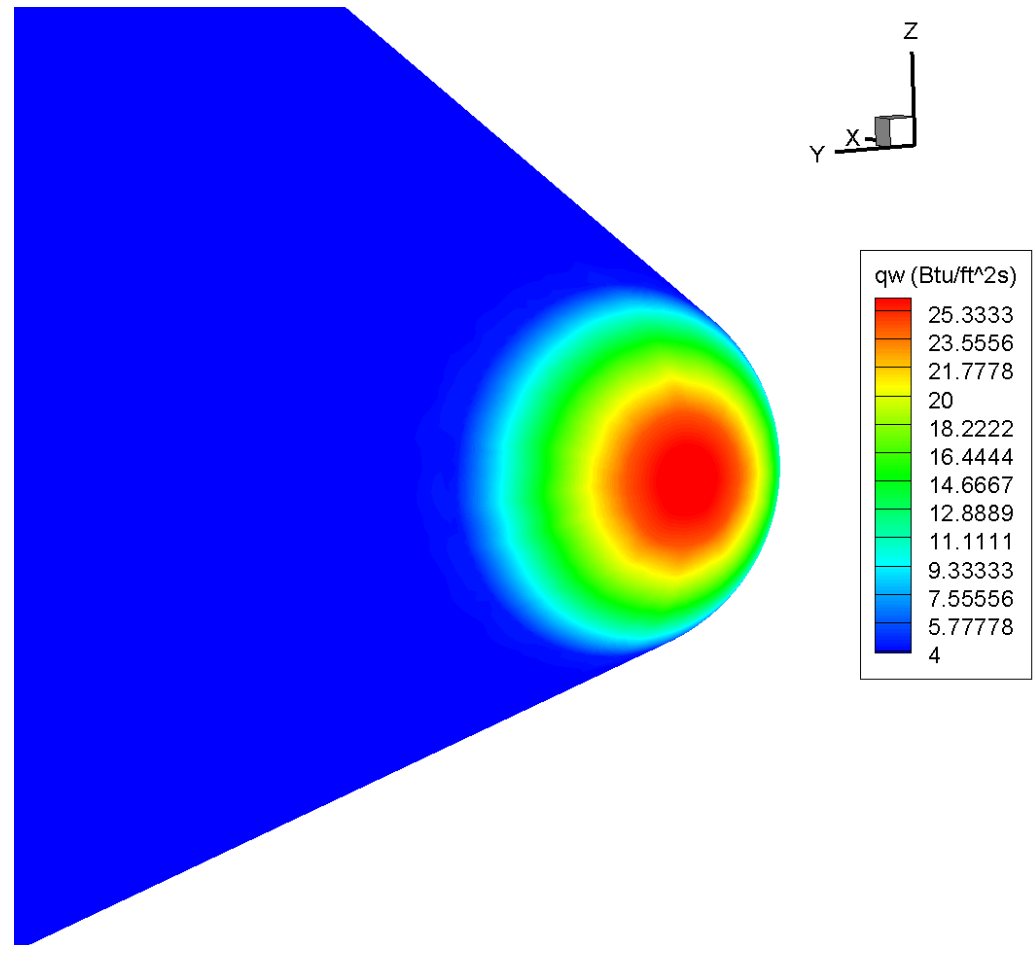

Figure 9: UNLATCH Surface Heating Solution for Wind-Tunnel case 
A case study was performed to compare the agreement of the solution derived from UNLATCH with experimental data. ${ }^{37}$ Convective heating measurements were taken with thermocouples embedded in the model in a wind-tunnel at Mach 10.6 for a 15-degree sphere-cone with 1.1 inch nose radius at constant wall temperature of $530^{\circ} R$ and $\alpha=0^{\circ}$. The procedure outlined in previously was used to iterate the inviscid flowfield mesh to capture an accurate heating solution.

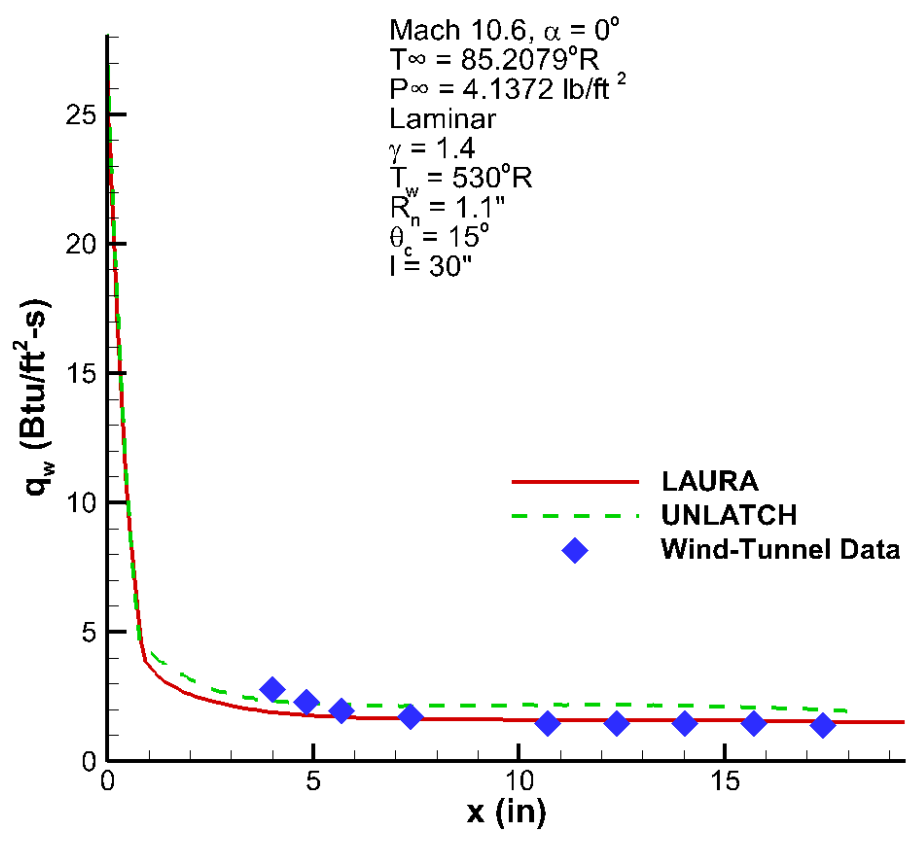

Figure 10: LAURA and UNLATCH Laminar Heating Prediction Comparison with WindTunnel Test Data

Figure 8 illustrates the inviscid solution. Pressure and density are displayed on the surface and in the flowfield respectively. The resulting surface heating distribution is well behaved as shown in Fig. 9. A benchmark LAURA solution was generated for comparison. Figure 10 compares the LAURA and UNLATCH symmetry plane (centerline) surface heating predictions to the experimental wind-tunnel data. Good agreement is shown between the two predictions and both compare well to the available wind-tunnel data, which starts at $x=3.937$ inches.

\section{Entry Trajectory Flight Envelope: Evaluating the Solution Space}

The near-term goal of this mid-fidelity tool implementation is to generate an aerothermal database that can be used to provide transient aeroheating environments to support TPS design and analysis. The database consists of heating solutions at an array of points within the entry trajectory flight envelope. As demonstrated in Section IV, accuracy of these heating solutions using the inviscid/boundary-layer method (mid-fidelity approach) is highly dependent upon the resolution of the inviscid flowfield mesh and solution, particularly in the stagnation region. However, generating each inviscid flowfield solution with sufficient resolution to yield accurate heating is the most time-consuming aspect of the mid-fidelity approach. Thus, populating the trajectory space with accurate heating solutions using the minimum number of flowfield solutions, is crucial to the rapid turn-around time desired during the conceptual design phase. This section explores the limitations associated with using a single flowfield solution at conditions in the neighborhood of, but not at the original trajectory point for which it was created. Flow condition parameters are varied independently to perform sensitivity studies on the heating solutions derived from flowfield generated at selected points along a trajectory profile. Heating solutions are compared with LAURA results for verification. 
All inviscid solutions are generated using the feature-based adaptation grid that uses the equilibriumair-chemistry flow solver, with radiation equilibrium temperature as wall boundary condition for the heating calculations. Radiation equilibrium temperature is the wall temperature reached when incoming heat is re-radiated away from the vehicle surface, depending on its emissivity. In general, assuming a highly insulative TPS (e.g. the Shuttle tiles), this value represents a good approximation of an entry vehicle's surface temperature. The $15^{\circ}$ sphere-cone at angle of attack of $20^{\circ}$ was used for the following studies.

\section{A. Approach}

Mission requirements and vehicle performance capability determine the limits of the entry flight envelope. When TPS requirements are considered, the lower edge of the flight trajectory envelope is bounded by a reference heating constraint (laminar) and the onset of turbulent heating. The upper limit is often represented by an "equilibrium glide" profile. The nominal mission is flown within these bounds, shown in Fig. 11(a). Representative heating constraints are identified in Fig. 11(b) and regions of peak laminar and turbulent heating levels are shown. The specific bounds are determined by the type of TPS. It is important to note that the trajectory profile is not only dependent on the altitude and velocity, but also on angle of attack, which is constrained by trim limits. For simplicity, a representative flight envelope is illustrated in Fig. 12.

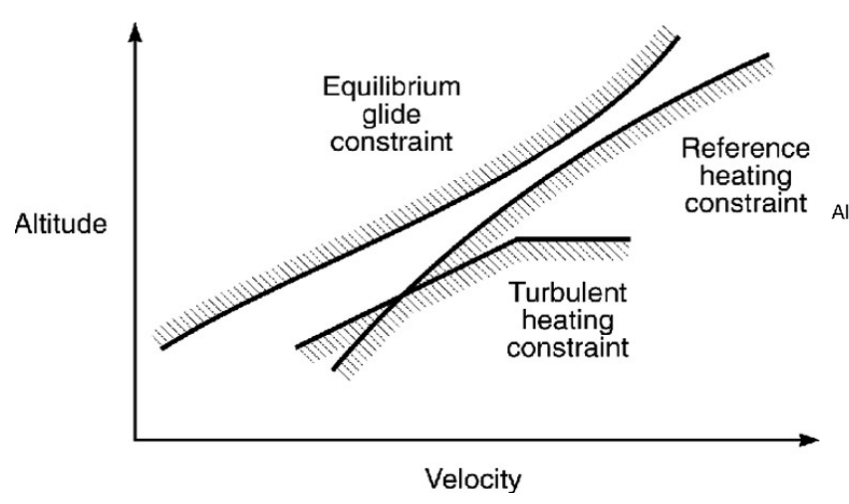

(a) Trajectory constraint for entry vehicles

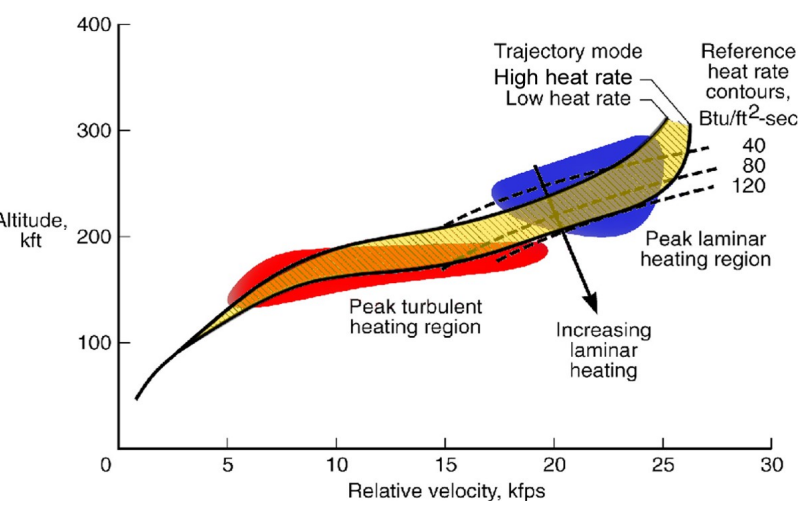

(b) Heating along entry trajectory envelope

\section{Figure 11: TPS-Related Entry Trajectory Constraints ${ }^{5}$}

A selected number of inviscid solutions are generated along the nominal flight profile. Inviscid solutions at the "baseline conditions", flight conditions for cases that lie on the flight profile, will be calculated. Another set of inviscid solutions will be calculated at the nearby flight conditions $( \pm H, \pm M, \pm \alpha)$, referred to as the "perturbed conditions". The heating solutions at the perturbed conditions are calculated from and compared for both sets of inviscid solutions. The $\alpha, H$, and $M$ sensitivities are assessed to gain insight into the acceptable perturbation limits from the nominal points and to determine the requirements for additional inviscid cases necessary to provide the backbone for the UNLATCH population of the aerothermal database. In the long term, run-time interpolation along any flight profile bounded by the pre-populated flight envelope, will provide transient environments necessary for TPS selection, design, and sizing at a conceptual level. Figure 12 illustrates a notional view of the trajectory envelope as populated in an altitude-velocity space. This view shows the potential for the use of a single CART3D solution to predict heating in its surrounding altitude-velocity space for as many as 13 UNLATCH solutions. For the purposes of this notional discussion, it is assumed that the AOA is constant over the range of altitudes and velocities considered. A good quality inviscid solution is critical as it will allow better prediction of the nearby space (perturbed cases). The limits of these perturbations where a good inviscid solution can predict acceptable heating is explored through sensitivity studies. Because the application of this mid-fidelity tool is intended mainly for conceptual work, a $20 \%$ bound on the heating levels is considered acceptable for these purposes.Altitude, Mach number and angle-of-attack sensitivities performed to date are presented.

A Shuttle entry trajectory profile was used in this investigation to examine the sensitivity to variations in altitude and Mach number is shown in Fig. 13. This profile was chosen because it is representative of a typical trajectory profile for lifting/winged bodies entering from LEO. It should be noted that the simple sphere-cone geometry used for this study would not have the aerodynamic control necessary to follow the 


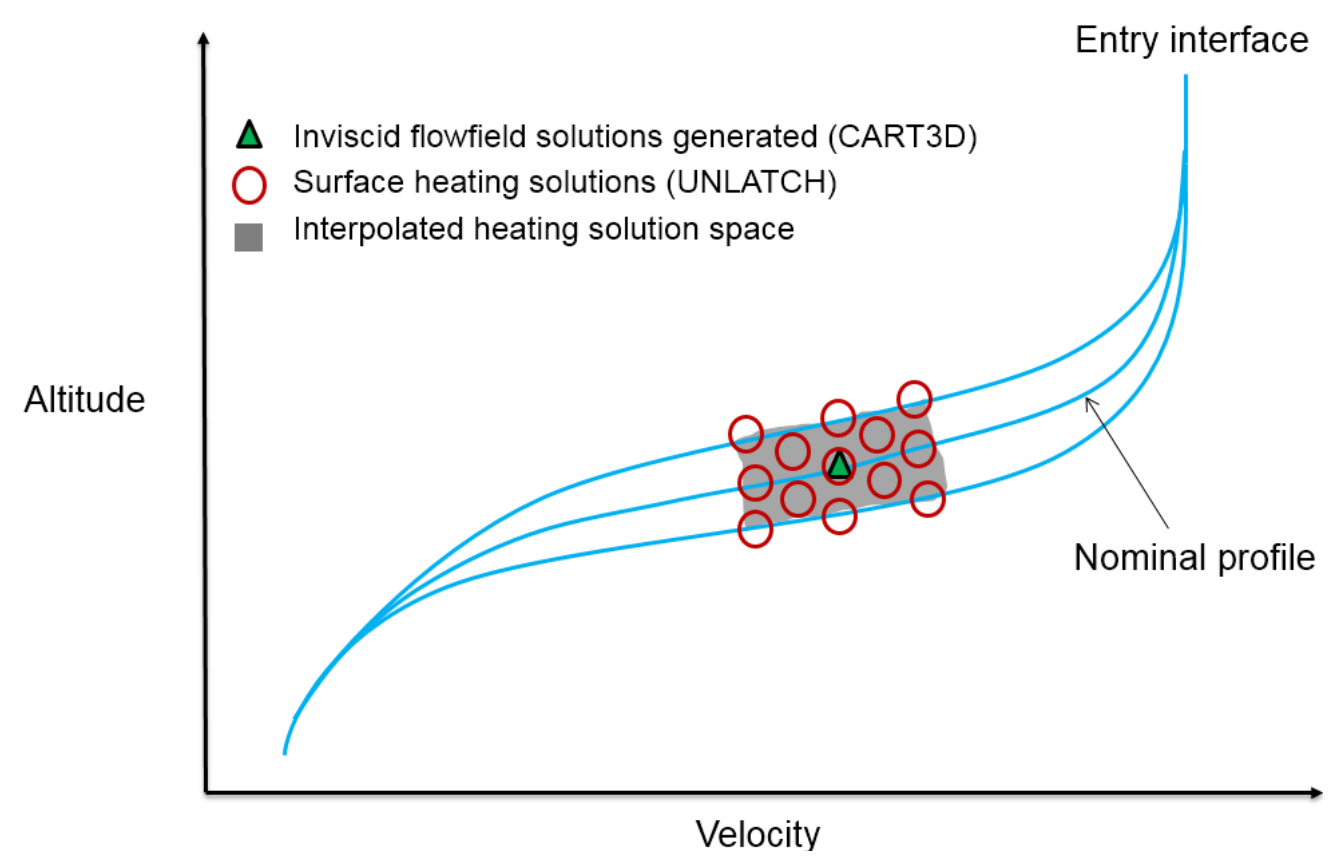

Figure 12: Strategy for populating trajectory envelope

trajectory profile depicted in Fig. 13. This trajectory does however provide a reasonable starting point for the establishment of a realistic flight envelope. Once established, the envelope is used to explore the extent to which a baseline inviscid flowfield solution (generated at a point on the nominal flight profile) can be used to successfully generate surface heating solutions at perturbed altitude, or Mach, conditions. This in turn will be used to inform the number of conditions at which inviscid flowfield solutions will be required to support the population of an aerothermal database.

\section{B. Sensitivity to Variation in Altitude}

The first trade study examines a variation in altitude off the nominal (baseline) condition for a given Mach number. Based on the STS-28 trajectory, ${ }^{38}$ together with the U.S. 1976 Standard Atmospheric Model, ${ }^{39}$ Mach numbers of interest and the associated altitudes were identified. Figure 13 shows the altitude-Mach representation of the trajectory points selected for this investigation and illustrates a representative sensitivity study variations in altitude and Mach number. The Mach 13.16 case, the green circle in Fig. 13, represents the original demonstration case. Other Mach numbers, 11 and 15, were selected due to their proximity to the original case for which the resultant surface heating solution has been verified, comparing well with the benchmark results from LAURA (Fig. 7) The Mach 18 condition was chosen to gain insight closer to a peak laminar heating condition, typically occurring near Mach 20. For each baseline condition, the altitude is varied $\pm 10,000 \mathrm{ft}$ and $\pm 20,000 \mathrm{ft}$ while the velocity is varied such that the freestream Mach number is consistent with that for which the baseline flowfield solutions was computed. Surface heating solutions were generated in two ways for each perturbed case: 1) the baseline inviscid flowfield solution calculated at the original trajectory point and 2) using the inviscid flowfield solution calculated at the perturbed altitude. Table 2 illustrates the freestream conditions for all altitudes considered in the trade space. The rows highlighted in blue represent the baseline conditions.

Results are not shown for the Mach 15 case, which brought to light an apparent coding issue in UNLATCH as evidenced by UNLATCH's inability to successfully resolve the stagnation region and compute along the streamlines. The inviscid solution was re-examined and confirmed to be of acceptable quality to compute heating and that inconsistencies in the velocities were unlikely to be the source of issue. The code developer has been made aware of the problem and is working to resolve. Inconsistent behavior for this case when using alternate computing platforms (eg. Linux, Windows, OSX) and/or compilers suggests memory overrun may be the root cause. To date, the problem has not been properly identified. Therefore, calculations for the Mach 15 cases were suspended and remain on hold, as were the $-20,000 \mathrm{ft}$ trial for the Mach 13.16 case 


\section{STS-28}

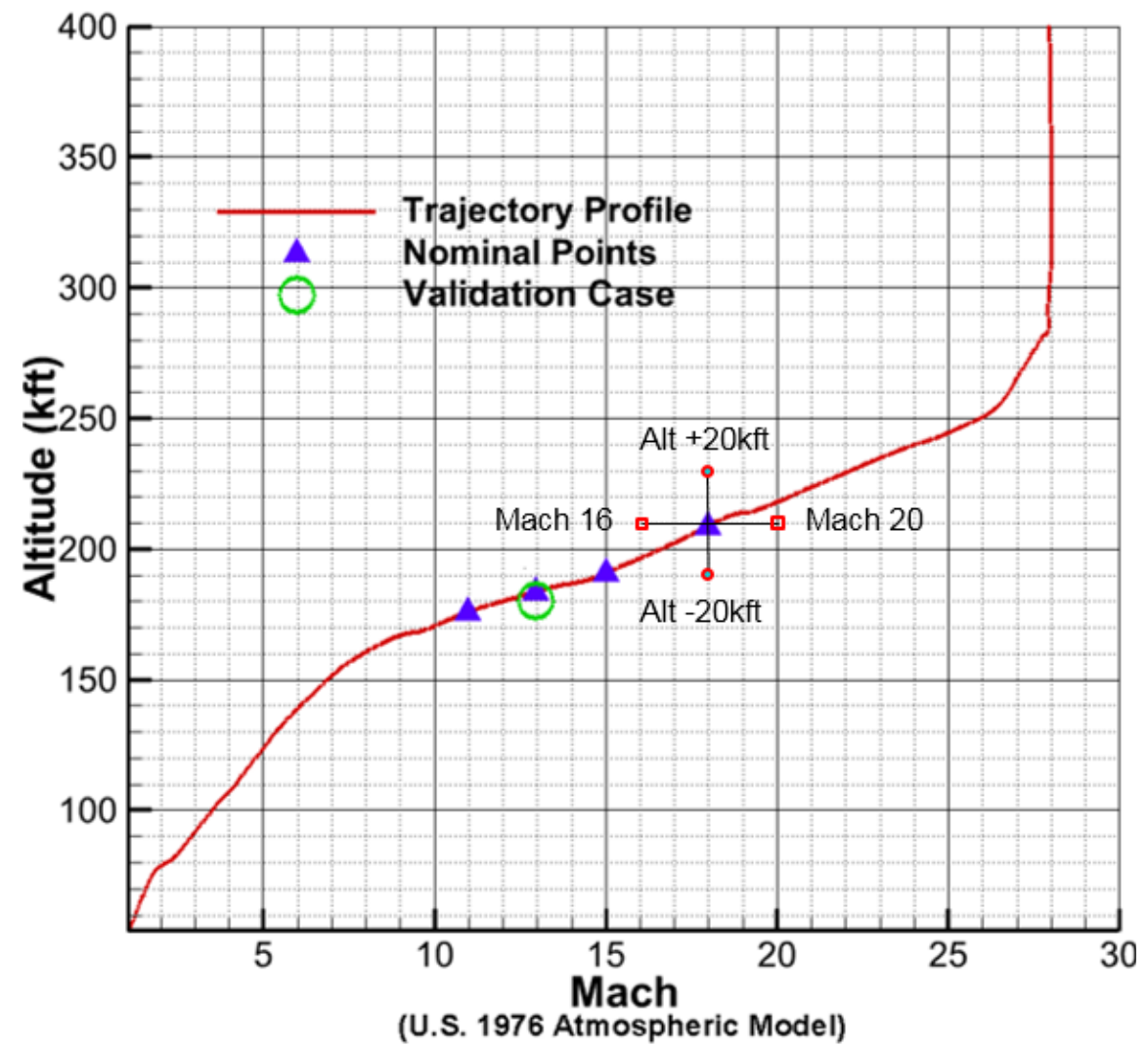

Figure 13: STS-28 entry trajectory profile and selected nominal points

Table 2: Freestream Flight Conditions Investigated (U.S. 76 Atmospheric Model)

\begin{tabular}{cccccccc}
\hline \hline & $\mathbf{H}$ & $\mathbf{V}_{\infty}$ & $\mathbf{T}_{\infty}$ & $\mathbf{P}_{\infty}$ & $\begin{array}{c}\boldsymbol{\rho}_{\infty} \\
\text { slugs } / f t^{3}\end{array}$ & $\begin{array}{c}\mathbf{a} \\
f t / s\end{array}$ & $\mathbf{M}$ \\
\hline 1 & 156,000 & 11902.2 & 487.170 & 2.2598 & $2.7 \times 10^{-6}$ & 1082.02 & 11 \\
2 & 166,000 & 11902.2 & 487.170 & 1.5471 & $1.8501 \times 10^{-6}$ & 1082.02 & 11 \\
3 & 176,000 & 11766.3 & 476.103 & 1.0562 & $1.2924 \times 10^{-6}$ & 1069.66 & 11 \\
4 & 186,000 & 11578.2 & 461.004 & 0.7128 & $9.0079 \times 10^{-7}$ & 1052.56 & 11 \\
5 & 196,000 & 11387.1 & 445.920 & 0.4750 & $6.21 \times 10^{-7}$ & 1035.19 & 11 \\
\hline 6 & 160,000 & 14239.4 & 487.17 & 1.9419 & $2.32 \times 10^{-6}$ & 1082.02 & 13.16 \\
7 & 170,000 & 14210 & 485.169 & 1.3230 & $1.5965 \times 10^{-6}$ & 1079.79 & 13.16 \\
8 & 180,000 & 13987.1 & 470.062 & 0.9038 & $1.12 \times 10^{-6}$ & 1062.85 & 13.16 \\
9 & 190,000 & 13760.8 & 454.969 & 0.6070 & $7.7717 \times 10^{-7}$ & 1045.65 & 13.16 \\
10 & 200,000 & 13530.72 & 439.89 & 0.4023 & $5.2379 \times 10^{-7}$ & 1028.17 & 13.16 \\
\hline 11 & 190,000 & 18821.7 & 454.969 & 0.6070 & $7.7717 \times 10^{-7}$ & 1045.65 & 18 \\
12 & 200,000 & 18507.1 & 439.890 & 0.4023 & $5.2379 \times 10^{-7}$ & 1028.17 & 18 \\
13 & 210,000 & 18187.4 & 424.826 & 0.2630 & $3.6062 \times 10^{-7}$ & 1010.41 & 18 \\
14 & 220,000 & 17862.4 & 409.776 & 0.1694 & $2.4076 \times 10^{-7}$ & 992.35 & 18 \\
15 & 230,000 & 17531.6 & 394.74 & 0.1073 & $1.58 \times 10^{-7}$ & 973.98 & 18 \\
\hline
\end{tabular}


(Case 6), and the $-20,000$ and $-10,000 \mathrm{ft}$ trials for the Mach 18 case (Case 11 and 12, respectively), all of which failed with the same apparent error as that encountered on the Mach 15 cases.

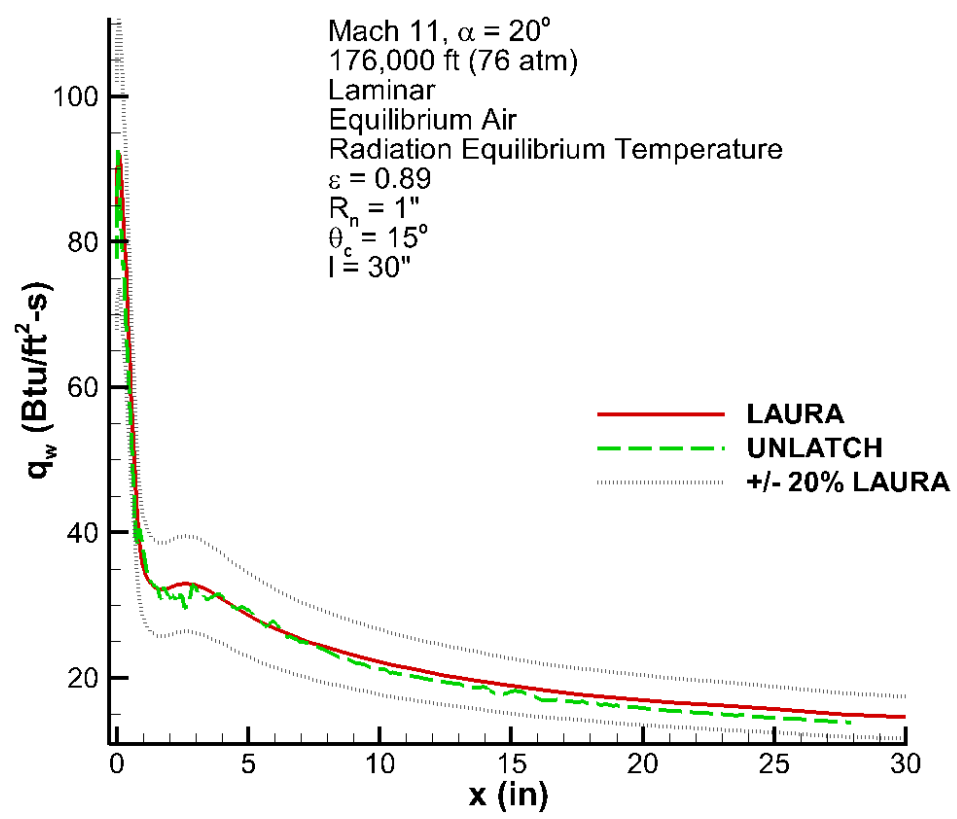

Figure 14: Benchmark windward centerline comparison for Mach 11 case at 176,000 ft (Case 3)

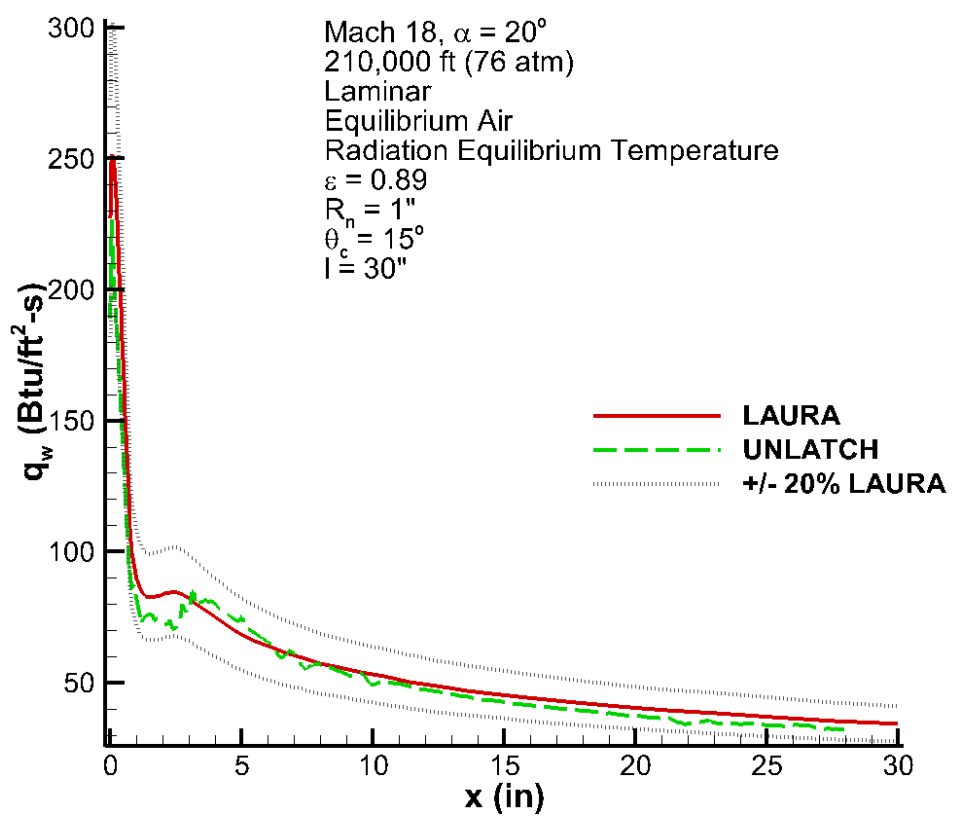

Figure 15: Benchmark comparison for Mach 18 case at 210,000 ft (Case 13) 
Overall, the baseline flowfield solutions (windward centerline heating plots shown in Fig. 14, 7, and 15, corresponding to Mach 11, 13.16, and 18 respectively) yielded heating solutions that were well within $20 \%$ of the LAURA benchmark solutions. Accuracy seems to be more difficult to achieve for the higher Mach numbers, despite adhering to the guidelines presented for an "ideal" inviscid solution. Generating accurate results for the stagnation and recompression areas of vehicle heating were particularly challenging. Good agreement with the benchmark solutions is noted (Fig. 14, 7, and 15), particularly downstream of the recompression region. Tables 3, 4, and 5 show the stagnation heating results obtained for the Mach 11, 13.16, and 18 trades respectively. From left to right, the columns contain the case number, the flight condition being modeled, the flight condition used for the flowfield (FF) solution and the associated heat rate, the flight condition of the baseline flowfield solution and the associated heat rate, and the LAURA benchmark solutions. The angle of attack for all cases is $20^{\circ}$. Again, the blue-highlighted rows represent the baseline cases.

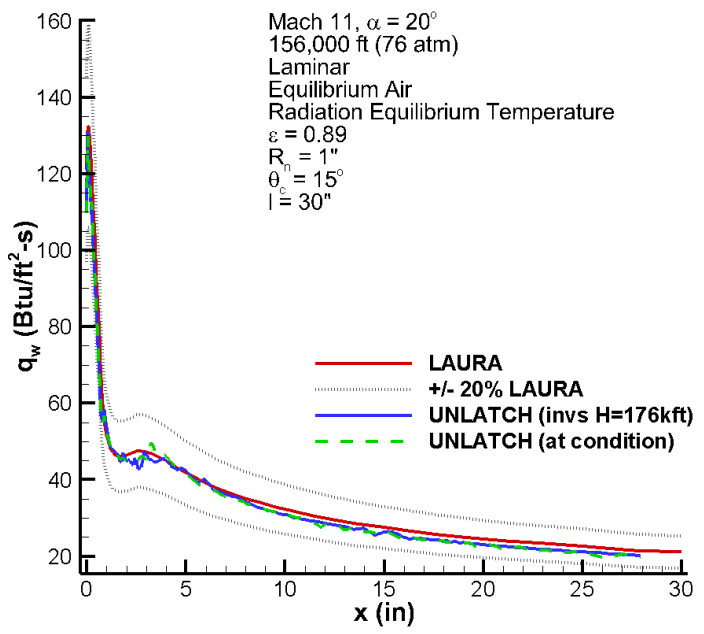

(a) $156,000 \mathrm{ft}$ (Case 1)

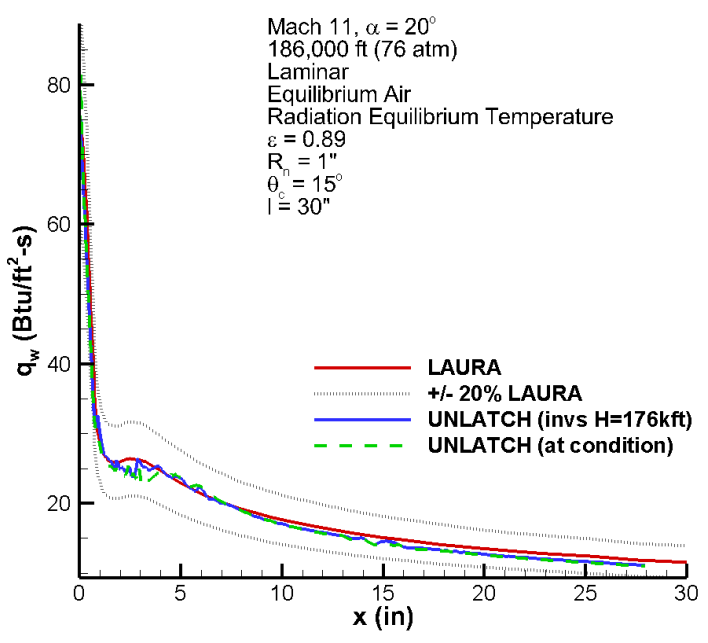

(c) $186,000 \mathrm{ft}$ (Case 4)

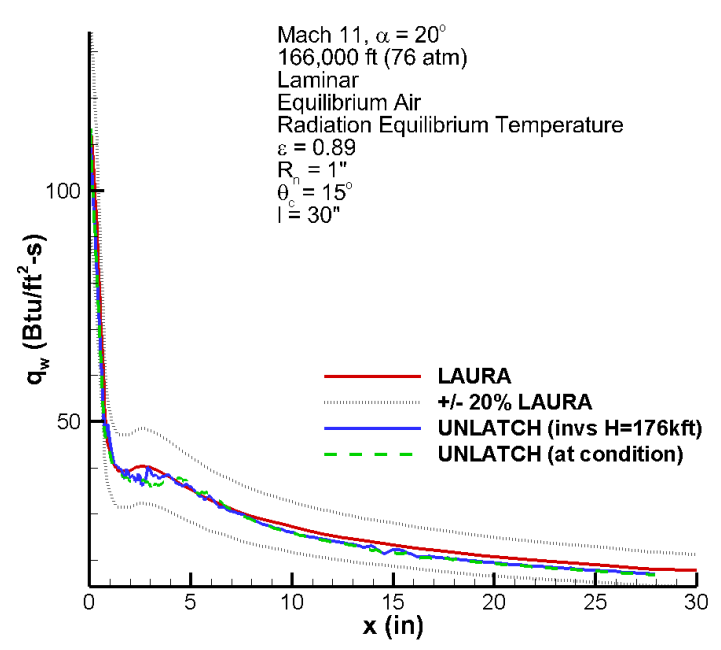

(b) $166,000 \mathrm{ft}$ (Case 2)

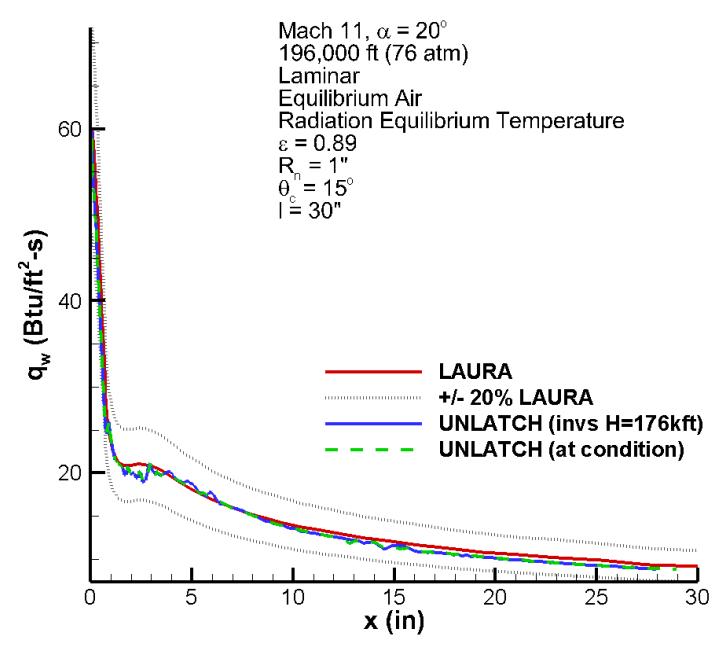

(d) $196,000 \mathrm{ft}$ (Case 5)

Figure 16: Benchmark comparison for Mach 11 variation in altitude using inviscid solution computed at $176 \mathrm{kft}$

For the Mach 11 case, Table 3, the UNLATCH solutions generated from the baseline condition for all perturbed conditions show good agreement in stagnation heating rates with those generated from the 
Table 3: Stagnation Point Heating at Mach 11: Altitude variation $\pm 10,000 \mathrm{ft}, \pm \mathbf{2 0 , 0 0 0} \mathrm{ft}$

\begin{tabular}{|c|c|c|c|c|c|c|}
\hline & Condition & \multicolumn{4}{|c|}{ UNLATCH Solutions } & LAURA \\
\hline Case & $\begin{array}{l}\mathbf{H} \\
f t\end{array}$ & $\begin{array}{c}\text { FF Sol'n } \\
\text { Adapted At: } \\
f t\end{array}$ & $\begin{array}{c}\mathbf{q}_{\mathbf{s}} \\
B t u / f t^{2} s\end{array}$ & $\begin{array}{c}\text { FF Sol'n } \\
\text { Adapted At: } \\
f t\end{array}$ & $\begin{array}{c}\mathbf{q}_{\mathbf{s}} \\
B t u / f t^{2} s\end{array}$ & $\begin{array}{c}\mathbf{q}_{\mathbf{s}} \\
B t u / f t^{2} s\end{array}$ \\
\hline 1 & 156,000 & 156,000 & 129.89 & 176,000 & 131.0 & 132.5 \\
\hline 2 & 166,000 & 166,000 & 112.09 & 176,000 & 112.2 & 111.94 \\
\hline 3 & 176,000 & 176,000 & 92.60 & 176,000 & 92.6 & 92.24 \\
\hline 4 & 186,000 & 186,000 & 74.29 & 176,000 & 74.92 & 74.00 \\
\hline 5 & 196,000 & 196,000 & 59.00 & 176,000 & 60.1 & 59.92 \\
\hline
\end{tabular}

perturbed condition inviscid solution. These heating solutions along the windward symmetry plane also agree in level and behavior with LAURA solutions, as shown in Fig. 16. For each case, the solid red line represents the LAURA solution, the blue line the UNLATCH solution using the baseline inviscid solution $(176 \mathrm{kft})$ and the green dashed line the UNLATCH solution with the inviscid solution computed at the perturbed condition. The results show that in this case, a single baseline inviscid solution can indeed be used with UNLATCH to accurately predict heating for a variation of up to $20 \mathrm{kft}$ in altitude.

For the Mach 13.16 case (Table 4), with the exception of case 6, the computed UNLATCH stagnation heating results for all cases show similar behavior between those using the inviscid solution of the baseline and those using the inviscid solution at the perturbed conditions. Agreement with LAURA benchmark heating along the centerline (not shown here) for this case was also noted; similar behavior to that illustrated in Fig. 16 for the Mach 13 case is observed. Case 6 is the $20 \mathrm{kft}$ decrease in altitude trial that could not be computed due to the code issue mentioned previously.

Table 4: Stagnation Point Heating at Mach 13.16: Altitude variation $\pm 10,000 \mathrm{ft}, \pm 20,000 \mathrm{ft}$

\begin{tabular}{|c|c|c|c|c|c|c|}
\hline & Condition & \multicolumn{4}{|c|}{ UNLATCH Solutions } & LAURA \\
\hline Case & $\begin{array}{l}\mathbf{H} \\
f t\end{array}$ & $\begin{array}{c}\text { FF Sol'n } \\
\text { Adapted At: } \\
f t\end{array}$ & $\begin{array}{c}\mathbf{q}_{\mathbf{s}} \\
B t u / f t^{2} s\end{array}$ & $\begin{array}{c}\text { FF Sol'n } \\
\text { Adapted At: } \\
f t\end{array}$ & $\begin{array}{c}\mathbf{q}_{\mathbf{s}} \\
B t u / f t^{2} s\end{array}$ & $\begin{array}{c}\mathbf{q}_{\mathbf{s}} \\
B t u / f t^{2} s\end{array}$ \\
\hline 6 & 160,000 & 160,000 & ERROR & 180,000 & ERROR & 244.64 \\
\hline 7 & 170,000 & 170,000 & 179.50 & 180,000 & 192.54 & 205.70 \\
\hline 8 & 180,000 & 180,000 & 157.48 & 180,000 & 157.48 & 163.77 \\
\hline 9 & 190,000 & 190,000 & 127.44 & 180,000 & 127.37 & 131.03 \\
\hline 10 & 200,000 & 200,000 & 101.89 & 180,000 & 101.99 & 104.40 \\
\hline
\end{tabular}

Table 5 shows the stagnation heating results for the Mach 18 cases. Where the baseline inviscid solution is used, stagnation heating rates for altitude increases of 10 and $20 \mathrm{kft}$ fall within $15 \%$ of the LAURA benchmark. The associated windward centerline results are shown in figs. 17(a) and 17(b). Downstream of the recompression area, levels are in good agreement with the LAURA benchmark regardless of inviscid solutions computed at baseline or perturbed condition. The recompression region is poorly predicted, but this is attributed to poor resolution in the inviscid solutions that could be improved with further refinement. Excluding the recompression area, the UNLATCH solutions generated are in agreement with the LAURA predictions, as shown in Fig. 17. The cases at $10 \mathrm{kft}$ and $20 \mathrm{kft}$ lower altitudes (case 11 and 12) failed due to the code issue identified earlier and therefore no assessment could be performed.

In this sensitivity study, the inviscid solutions of the baseline/nominal points on the trajectory are used to predict heating at altitudes varied by $10 \mathrm{kft}$ and $20 \mathrm{kft}$, holding Mach number constant. The heating solutions generated using the inviscid solutions at the baseline condition for all successful cases generally showed consistent behavior with those generated using inviscid solutions at the perturbed conditions. This study shows that it is possible to predict heating using the baseline inviscid solution of a point on the trajectory profile for an altitude variation of up to $20 \mathrm{kft}$. 
Table 5: Stagnation Point Heating at Mach 18: Altitude variation $\pm 10,000 \mathrm{ft}, \pm \mathbf{2 0 , 0 0 0} \mathrm{ft}$

\begin{tabular}{|c|c|c|c|c|c|c|}
\hline & Condition & \multicolumn{4}{|c|}{ UNLATCH Solutions } & LAURA \\
\hline Case & $\begin{array}{l}\mathbf{H} \\
f t\end{array}$ & $\begin{array}{c}\text { FF Sol'n } \\
\text { Adapted At: } \\
f t\end{array}$ & $\begin{array}{c}\mathbf{q}_{\mathbf{s}} \\
B t u / f t^{2} s\end{array}$ & $\begin{array}{c}\text { FF Sol'n } \\
\text { Adapted At: } \\
f t\end{array}$ & $\begin{array}{c}\mathbf{q}_{\mathbf{s}} \\
B t u / f t^{2} s\end{array}$ & $\begin{array}{c}\mathbf{q}_{\mathbf{s}} \\
B t u / f t^{2} s\end{array}$ \\
\hline 11 & 190,000 & 190,000 & ERROR & 210,000 & ERROR & 408.89 \\
\hline 12 & 200,000 & 200,000 & ERROR & 210,000 & ERROR & 320.05 \\
\hline 13 & 210,000 & 210,000 & 226.86 & 210,000 & 226.86 & 253.66 \\
\hline 14 & 220,000 & 220,000 & 165.33 & 210,000 & 179.23 & 198.52 \\
\hline 15 & 230,000 & 230,000 & 140.41 & 210,000 & 135.02 & 162.03 \\
\hline
\end{tabular}

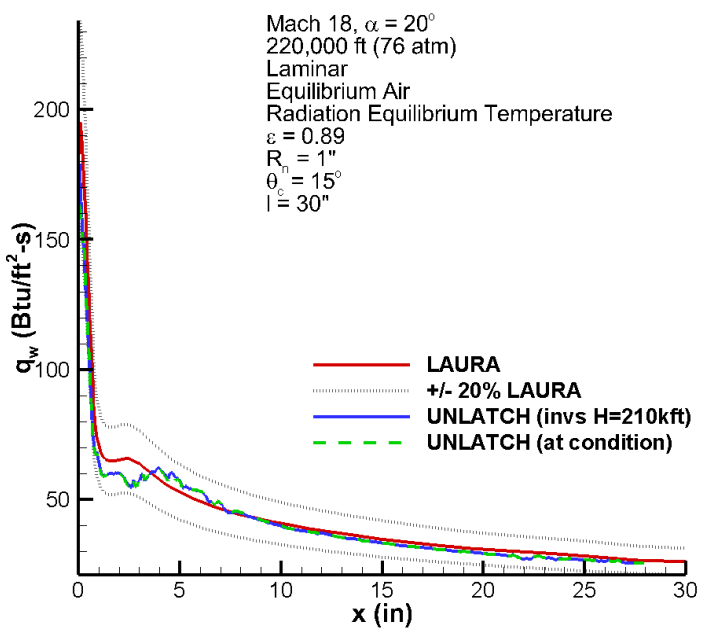

(a) 220,000 ft (Case 14)

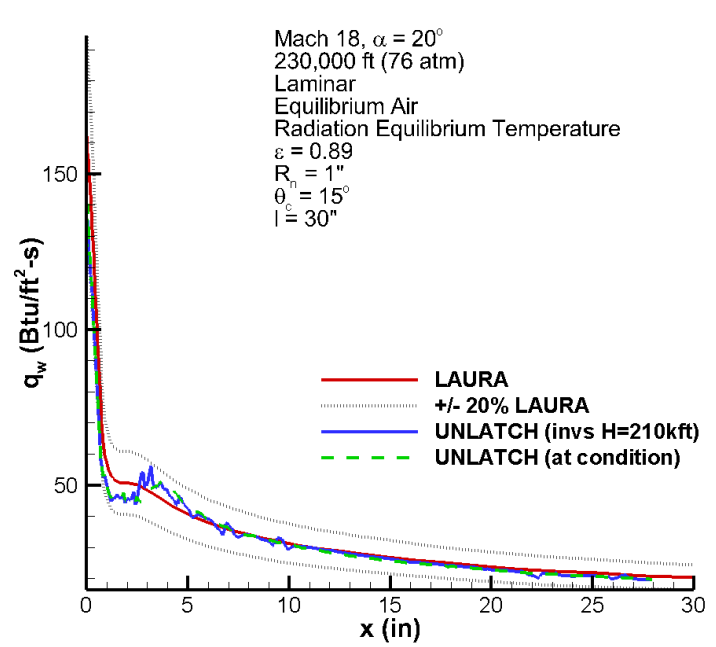

(b) 230,000 ft (Case 15)

Figure 17: Benchmark comparison for Mach 18 variation in altitude using inviscid solution at $210 \mathrm{kft}$

\section{Sensitivity to Variation in Mach Number}

This section focuses on the applicability of a baseline flowfield solution at nearby Mach numbers. The altitude for each baseline case is held constant as the Mach number is varied by two. For instance, the baseline inviscid solution adapted at Mach 18 and 210,000 ft is used to calculate the surface heating for Mach 16 and Mach 20 cases at the same altitude (see Fig. 13). Another set of UNLATCH solutions are calculated using the inviscid solutions generated at the perturbed conditions (Mach 16 and 20) for comparison. Table 6 outlines the trade setup. The rows highlighted in blue represent the baseline flowfield solutions presented previously in Fig. 14, 7, and 15, where they were shown to agree favorably with the benchmark Navier-Stokes results. The two columns on the right show the perturbed conditions to be modeled.

Tables 7-9 compare the stagnation point heating results for this sensitivity study for baseline conditions Mach 11, 13.16 and 18 respectively. Similar to the table setup for the altitude variation, the condition that is modeled is listed in the second column from the left. The inviscid solution used and the associated stagnation heating solution calculated based on it are shown in the following columns, first using the inviscid calculation at the perturbed condition, followed by the results using the inviscid solution calculated at the original baseline condition. The last column shows the LAURA prediction for comparison.

Table 7 shows the stagnation point heating for the Mach number variation using Mach 11 at the $176 \mathrm{kft}$ as the baseline condition. These heating predictions using the inviscid solutions at the baseline and perturbed conditions are well within $10 \%$ of the LAURA benchmark. Figure 18 shows the associated centerline heating 
Table 6: Mach number variation trade setup

\begin{tabular}{cc|cc}
\hline \hline \multicolumn{2}{c|}{ Conditions FF Sol'n Adapted At: } & \multicolumn{2}{|c}{ Conditions Modeled } \\
\hline $\mathbf{M}$ & $\mathbf{H}$ & $\mathbf{M}$ & $\mathbf{H}$ \\
& $f t$ & & $f t$ \\
\hline 11 & 176,000 & 9 & 176,000 \\
& & 13.16 & 176,000 \\
\hline 13.16 & 11 & 180,000 \\
& 180,000 & 15 & 180,000 \\
\hline 18 & & 16 & 210,000 \\
& 210,000 & 20 & 210,000 \\
\hline
\end{tabular}

Table 7: Stagnation Point Heating at 176kft: Mach Number variation

\begin{tabular}{c|c|cc|cc|c}
\hline \hline & Condition & \multicolumn{4}{|c|}{ UNLATCH Solutions } & LAURA \\
\hline Case & $\mathbf{M}$ & $\begin{array}{c}\text { FF Sol'n } \\
\text { Adapted At: }\end{array}$ & $\begin{array}{c}\mathbf{q}_{\mathbf{s}} \\
B t u / f t^{2} s\end{array}$ & $\begin{array}{c}\text { FF Sol'n } \\
\text { Adapted At: }\end{array}$ & $\begin{array}{c}\mathbf{q}_{\mathbf{s}} \\
B t u / f t^{2} s\end{array}$ & $\begin{array}{c}\mathbf{q s}_{\mathbf{s}} \\
B t u / f t^{2} s\end{array}$ \\
\hline 1 & 9 & 9 & 41.88 & 11 & 43.93 & 43.57 \\
2 & 11 & 11 & 92.60 & 11 & 92.60 & 92.24 \\
3 & 13.16 & 13.16 & 161.95 & 11 & 174.01 & 178.94 \\
\hline
\end{tabular}

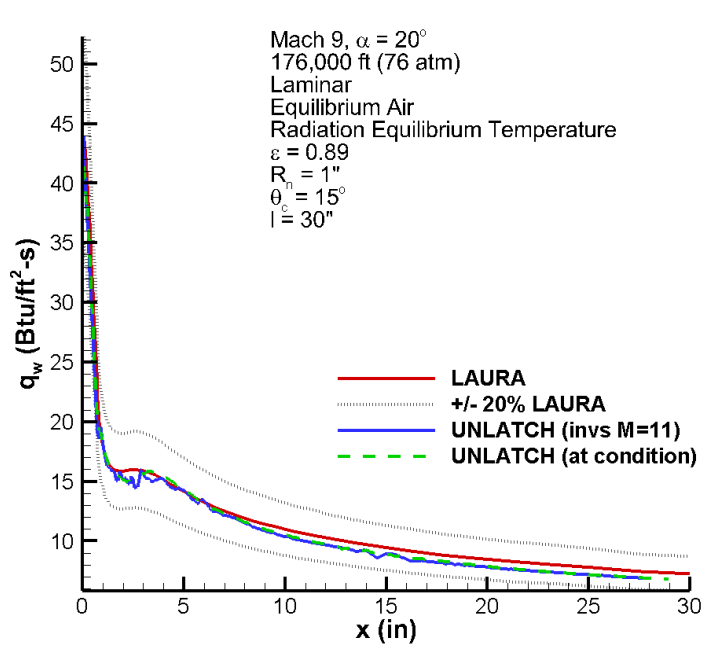

(a) Mach 9

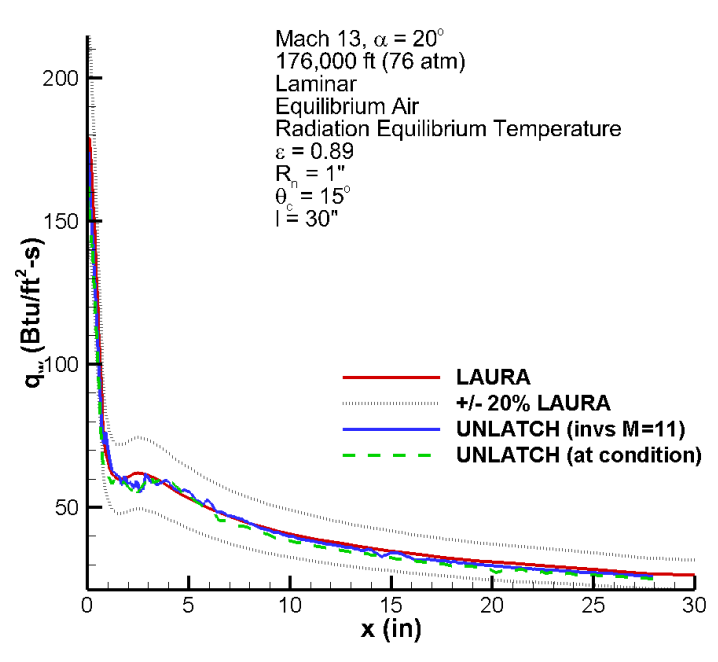

(b) Mach 13

Figure 18: Benchmark comparison for variation in Mach number using the inviscid solution at Mach 11, 176kft

distribution predicted along the windward centerline. The solid red line represents the LAURA heating solution, the solid blue line shows the UNLATCH solution calculated with the baseline inviscid solution, and the green dashed line represents the UNLATCH solution obtained using the perturbed inviscid solution. Figure 18 illustrates the surface heating solutions, corresponding to the stagnation predictions shown in Table 7. In Fig. 18(a), the Mach 9 heating solution generated with the Mach 11 inviscid solution is shown to compare well with that generated with the Mach 9 inviscid solution and both are well within $20 \%$ of the LAURA benchmark solution. Similarly, the Mach 13.16 heating solutions generated with the inviscid solution at Mach 11 and 13.16 are shown in Fig. 18(b). Both show good agreement with respect to each 
other and also to the LAURA prediction. In each case, heating shows similar behavior using the inviscid solution at either the baseline or the perturbed condition.

Table 8: Stagnation Point Heating at 180kft: Mach Number variation

\begin{tabular}{|c|c|c|c|c|c|c|}
\hline & Condition & \multicolumn{4}{|c|}{ UNLATCH Solutions } & LAURA \\
\hline Case & $\mathbf{M}$ & $\begin{array}{c}\text { FF Sol'n } \\
\text { Adapted At: }\end{array}$ & $\begin{array}{c}\mathbf{q}_{\mathbf{s}} \\
B t u / f t^{2} s\end{array}$ & $\begin{array}{c}\text { FF Sol'n } \\
\text { Adapted At: }\end{array}$ & $\begin{array}{c}\mathbf{q}_{\mathbf{s}} \\
B t u / f t^{2} s\end{array}$ & $\begin{array}{c}\mathbf{q}_{\mathbf{s}} \\
B t u / f t^{2} s\end{array}$ \\
\hline 4 & 11 & 11 & 85.08 & 13.16 & 83.63 & 84.91 \\
\hline 5 & 13.16 & 13.16 & 157.48 & 13.16 & 157.48 & 163.77 \\
\hline 6 & 15 & 15 & ERROR & 13.16 & ERROR & 266.57 \\
\hline
\end{tabular}

Figures are not shown for similar trials that were conducted using the Mach 13.16 baseline for predictions at Mach 11 and Mach 15. The Mach 11 case predictions showed good agreement regardless of which inviscid solution was used. No results were obtained for the Mach 15 case due to the UNLATCH issue noted previously. Stagnation heating predictions are displayed in Table 8, showing agreement well within $10 \%$ for the Mach 11 prediction (case 4) compared to the heating solutions using the perturbed inviscid solution and LAURA benchmark. Again, no results is shown for the Mach 15 case due to the code issue in UNLATCH.

Table 9: Stagnation Point Heating at 210kft: Mach Number variation

\begin{tabular}{|c|c|c|c|c|c|c|}
\hline \multirow[b]{2}{*}{ Case } & \multirow{2}{*}{$\begin{array}{c}\text { Condition } \\
\text { M }\end{array}$} & \multicolumn{4}{|c|}{ UNLATCH Solutions } & \multirow{2}{*}{$\begin{array}{c}\text { LAURA } \\
\mathbf{q}_{\mathbf{s}} \\
B t u / f t^{2} s\end{array}$} \\
\hline & & $\begin{array}{c}\text { FF Sol'n } \\
\text { Adapted At: }\end{array}$ & $\begin{array}{c}\mathbf{q}_{\mathbf{s}} \\
B t u / f t^{2} s\end{array}$ & $\begin{array}{c}\text { FF Sol'n } \\
\text { Adapted At: }\end{array}$ & $\begin{array}{c}\mathbf{q}_{\mathbf{s}} \\
B t u / f t^{2} s\end{array}$ & \\
\hline 7 & 16 & 16 & 154.46 & 18 & 149.37 & 168.47 \\
\hline 8 & 18 & 18 & 226.86 & 18 & 226.86 & 253.66 \\
\hline 9 & 20 & 20 & 300.22 & 18 & 121.45 & 387.55 \\
\hline
\end{tabular}

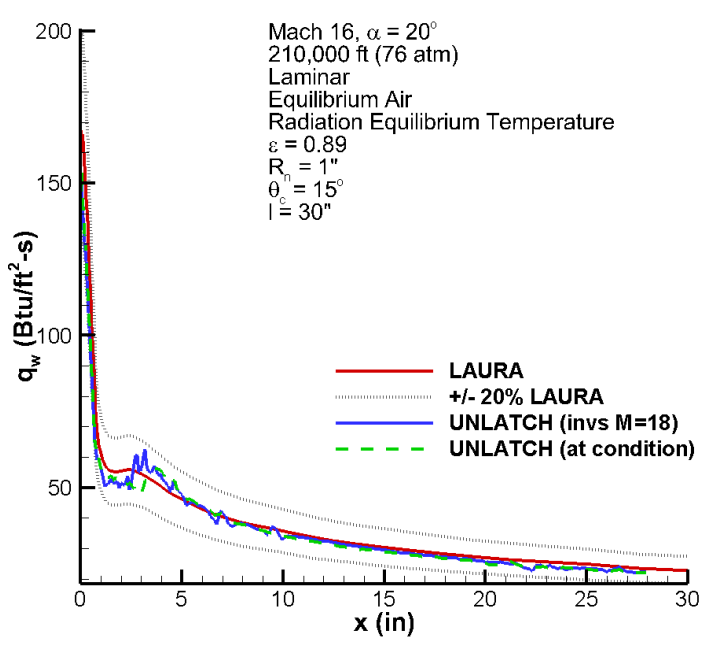

(a) Mach 16

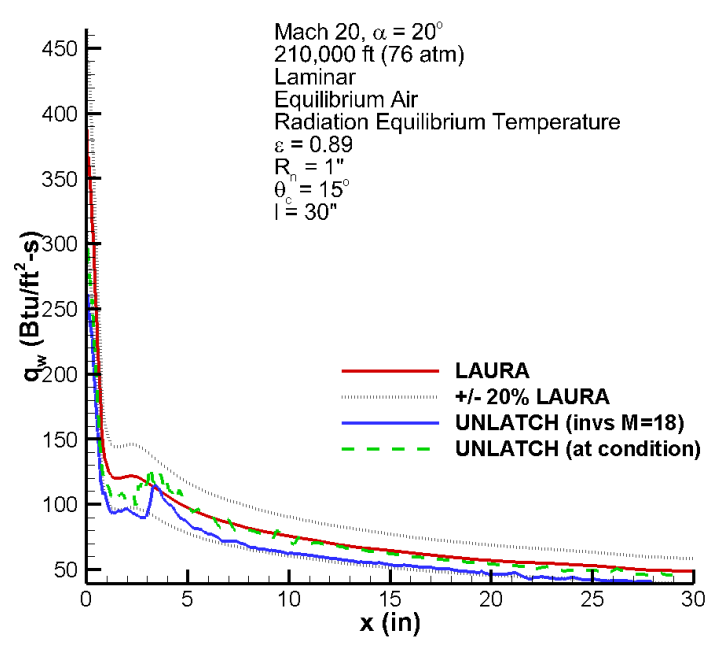

(b) Mach 20

Figure 19: Benchmark comparison for variation in Mach number using the inviscid solution at Mach 18, 210kft

Figure 19 illustrates the surface heating solutions corresponding to the stagnation heating predictions shown in Table 9 for the Mach 18 cases. Stagnation heating shown for the Mach 16 case, using the Mach 16 
and Mach 18 inviscid solutions, appears to be well within $20 \%$ of the LAURA benchmark. The centerline heating solutions of the Mach 16 case (see Fig.19(a)) also shows good agreement with the benchmark for both inviscid solutions (Mach 16 and Mach 18) aft of the recompression. However, the Mach 20 stagnation heating prediction using the Mach 18 inviscid solution showed a significant error (close to 70\%) from the LAURA benchmark. As observed in Fig. 19(b), the overall behavior of the Mach 20 case (using the Mach 18 and Mach 20 inviscid solutions) appears to be consistent with the LAURA benchmark. The heating levels of the Mach 20 UNLATCH solution using the baseline inviscid solution (Mach 18), however, appear to lay about $20 \%$ below the LAURA benchmark aft of the recompression region. In both cases (cases 7 and 9), significant noise is noted in the heating predictions, particularly in the recompression region. A closer examination of the inviscid solution showed the surface pressure to be the likely root of the noise. For the Mach 20 case (Fig. 19(b)), the UNLATCH solution relying on the inviscid solution at the perturbed condition also shows good agreement aft of the compression, although noise is again evident in the solution. For the case where the baseline Mach 18 inviscid solution is used instead, the heating solution is low by nearly $20 \%$ over the same region aft of the compression. This difference is attributed once again to the Mach 18 inviscid solution, which appears not to have adequate resolution in the shock layer.

This sensitivity study shows that using an inviscid solution to predict heating at the nearby Mach numbers (holding the altitude constant) is likely feasible. With the exception of the failed case at Mach 15, the UNLATCH heating results generated from the two sets of inviscid solutions closely matched the behavior and are generally in agreement to well within 20\%. Despite apparent noise in the Mach 18 inviscid solution, as well as shock layer resolution inadequate for the Mach 20 condition, the solutions still fall within this $20 \%$ bound. Additional refinement of the inviscid solution would be expected to resolve the recompression region more accurately, and to improve the agreement to within 10-15\% of the benchmark. A Mach number variation of two is likely feasible for applying an inviscid solution to generate heating solutions in its nearby Mach space, keeping the altitude constant. However, more studies are needed to explore the Mach number increment limitations, particularly at the higher Mach numbers.

\section{Sensitivity to Variation in Angle of Attack}

The objective of the angle of attack sensitivity study is to explore the applicability of the "baseline" inviscid flowfield solution to perturbed angle of attack conditions. The demonstration case $\left(\mathrm{M}=13.16, \alpha=20^{\circ}, 15^{\circ}\right.$ sphere-cone) is used. The angle of attack was varied $\pm 5^{\circ}$ from the nominal $20^{\circ}$ case. The flight condition from Table 1 is used for all solutions. Inviscid flowfield solutions are first computed explicitly for the perturbed angle-of-attack conditions, $15^{\circ}$ and $25^{\circ}$. The centerline heating distributions for the UNLATCH solutions generated in this manner are illustrated in Fig. 20. As expected, the heating is observed to increase with the angle of attack.

Table 10: Stagnation Point Heating at Mach 13.16: Angle of attack variation $\pm 5^{\circ}$

\begin{tabular}{c|cc|cc|c}
\hline \hline Condition & \multicolumn{4}{|c|}{ UNLATCH Solutions } & Benchmark \\
\hline & $\begin{array}{c}\text { FF Sol'n } \\
\text { Adapted At: } \\
\text { degrees }\end{array}$ & $\begin{array}{c}\mathbf{q}_{\mathbf{s}} \\
\text { Btu/ft} t^{2} s\end{array}$ & $\begin{array}{c}\text { FF Sol'n } \\
\text { Adapted At: } \\
\text { degrees }\end{array}$ & $\begin{array}{c}\mathbf{q}_{\mathbf{s}} \\
B t u / f t^{2} s\end{array}$ & $\begin{array}{c}\text { LAURA } \\
B t u / f t^{2} s\end{array}$ \\
\hline 15 & 15 & 141.2 & 20 & 157.5 & 163.768 \\
20 & 20 & 157.5 & 20 & 157.5 & 163.768 \\
25 & 25 & 161.9 & 20 & 157.5 & 163.768 \\
\hline
\end{tabular}

UNLATCH solutions are then generated for the perturbed angle of attack cases $\left(\alpha=15^{\circ}\right.$ and $\left.20^{\circ}\right)$ using the baseline inviscid flowfield solution computed for $\alpha=20^{\circ}$ case. The UNLATCH solutions computed explicitly at the perturbed angle of attack are compared to those computed using the baseline angle-of-attack flowfield solution, and also against the benchmark LAURA solutions at the corresponding angle-of-attack. Table 10, summarizes the stagnation point heating rates calculated with flowfield solutions that are adapted to different $\alpha$ cases. From left to right, the columns contain the $\alpha$ being modeled, the $\alpha$ used for the flowfield solution and the associated stagnation heating rate, the baseline flowfield solution and the associated heating rate, and finally the LAURA benchmark predicted heat rate. The highlighted row represents the baseline case. 


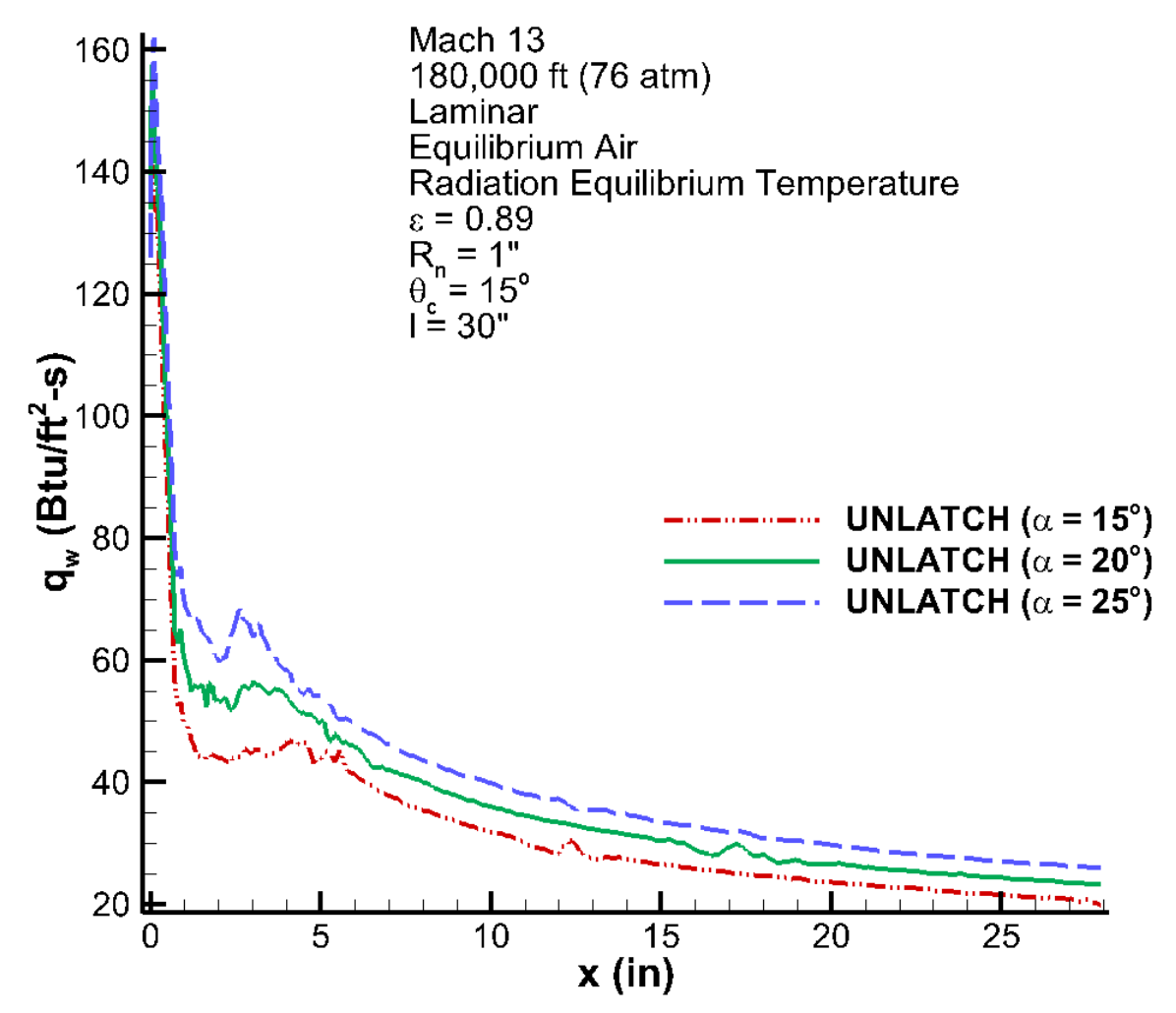

Figure 20: UNLATCH solutions for varying $\alpha$ using the flowfield solutions associated with each $\alpha$

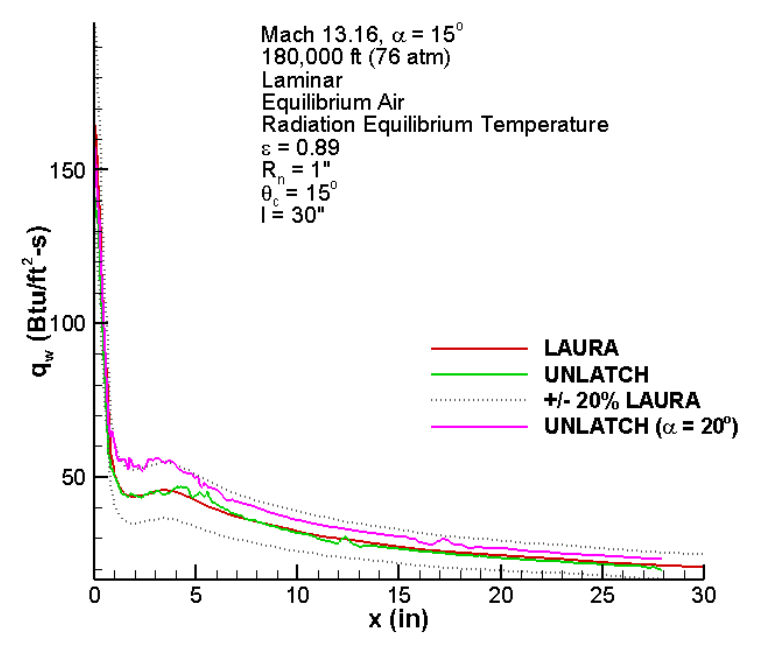

(a) $\alpha=15$

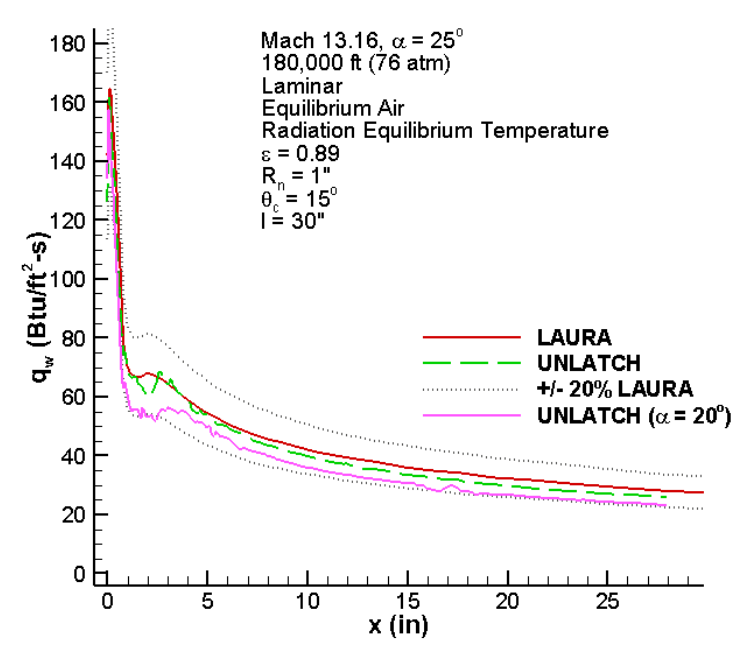

(b) $\alpha=25$

Figure 21: UNLATCH and LAURA solution comparisons at $\alpha=15^{\circ}$ and $25^{\circ}$

As expected, the LAURA stagnation point heating results, $163.768 \mathrm{Btu} / \mathrm{ft}^{2}$-s do not change with this small angle-of-attack variation, as the stagnation point remains on the spherical nosecap. UNLATCH solutions 
using the $20^{\circ}$ inviscid solution agreed identically with the unchanged stagnation point heating on the nose. The stagnation point heating is used simply as a measure of the level of heating exhibited in the UNLATCH cases compared to LAURA. However, the more important measure is the behavior of the solution in the windward symmetry plane and over the surface of the sphere cone. Figures 21(a) and 21(b) illustrate the centerline heating rates for the $15^{\circ}$ and $25^{\circ}$ cases respectively, shown in Table 10, as functions of axial distance along the cone. The benchmark LAURA solutions are identified by the red line and marked with a $20 \%$ band, within which the UNLATCH solutions are expected to fall. The UNLATCH solutions computed using the inviscid solutions for the actual angles of attack are shown in green (dashed line) and those computed using the baseline inviscid solution, in purple (dash dot). In both cases, the general behavior of the UNLATCH predictions for the centerline heat rate is consistent with the benchmark LAURA prediction. Both fall within the $20 \%$ bound considered acceptable for this mid-fidelity approach; the $15^{\circ}$ case near the upper bound and the $25^{\circ}$ case near the lower. However, this represents a poorer match than the other sensitivities have shown. Therefore, for consistent application to the aerothermal database development, it is recommended that increments smaller than the $5^{\circ}$ chosen for this study be explored. Similar studies should be performed at additional Mach numbers and angles of attack to provide the insight required to establish acceptable angle-of-attack increments over an expanded region of the flight envelope.

\section{E. Sensitivity Study Summary}

Sensitivity studies were performed to better understand the limits to using the mid-fidelity tool to predict heating in the nearby altitude and Mach number space, given a subset of a simplified trajectory profile. Study results demonstrated that it is possible to predict heating using the nominal baseline inviscid solution for up to a variation of $20 \mathrm{kft}$ in altitude and 2 in Mach number. A limited angle-of-attack study was also performed, showing that a variation of $5^{\circ}$ at $20^{\circ}$ angle of attack yields solutions that are within the $20 \%$ bound of the LAURA benchmark. However, a smaller increment should be considered in future studies as this result represents a poorer match than other sensitivities demonstrated here, generally within 10-15\%. Based on the results of the sensitivity studies performed to date, the number of inviscid solutions required to populate an aerothermal database for Mach numbers ranging from 9 to 20 and altitude variations of up to $20 \mathrm{kft}$, may be reduced to only a handful of solutions. Further study will be required to demonstrate that the same is true for a decrease in altitude.

\section{Concluding Remarks}

A mid-fidelity aeroheating tool, UNLATCH, was investigated for implementation in a multidisciplinary analysis environment to support TPS design and analysis for conceptual vehicle designs. An important motivation for this study was to enable rapid population of an aerothermal database within a desired flight envelope. The inviscid/boundary-layer approach explored in this paper has the potential to significantly reduce resource requirements compared to those for a Navier-Stokes solution-based aerothermal database. The tool uses the inviscid-flow/boundary-layer approach to combine a 3D inviscid flowfield solution and a boundary-layer approximation method to obtain heating solutions over an unstructured surface grid. The inviscid solver CART3D was used to generate the required flowfield solutions. A high-quality flowfield solution was found to be critical to obtaining an accurate heating solution with UNLATCH. In addition to satisfying the criteria for both residual convergence and a well-resolved pressure distribution in the flowfield solution, at least 10-20 volume cells must be packed within the shock layer to attain good resolution. Because UNLATCH calculations begin in the stagnation region and proceed along the streamlines, well-resolved flow around the stagnation area is particularly important; accuracy of the heating solutions is extremely sensitive to the small velocities present near the stagnation point. Representative UNLATCH solutions were validated against both Navier-Stokes solutions and experimental wind-tunnel data, and shown to compare well in each case.

The second phase of the study focused on gaining the insight required to formulate the population of an aerothermal database that would support the simulation of transient aeroheating environments for TPS analysis and design. Given the initial understanding of the procedures required to obtain good quality inviscid solutions and the associated heating as described previously, the investigation was expanded to determine the extent to which a single inviscid solution could be used to spawn multiple surface heating solutions. This was accomplished by varying the flight conditions, i.e. altitude, Mach and angle of attack, the parameters 
chosen to define the trajectory space. A simplified flight envelope was defined and UNLATCH solutions generated for selected baseline points within that trajectory space. The altitude-sensitivity studies performed demonstrated that, for the profile considered, it is possible to predict heating for a variation of up to 20 $\mathrm{kft}$, using the baseline inviscid solution. Similarly, within the Mach number range investigated, the study showed that it is possible to predict surface heating for a variation of \pm 2 in Mach number, using the baseline solution. If this holds, it suggests a significant reduction in the number of inviscid cases required to populate an aerothermal database. In both the altitude and the Mach number assessments, the heating solutions fell well within the recommended $20 \%$ bound (generally within approximately $10 \%$ ) of the benchmark solution. A limited sensitivity assessment was performed for angle-of-attack. Heating for variation of $\pm 5^{\circ}$ in angle of attack was studied for the Mach 13.16, $\alpha=20^{\circ}$, baseline case. Although the comparisons with benchmark solutions were just barely within the $20 \%$ bound, it is recommended that a smaller increment in angle of attack, $2^{\circ}$, be considered in future studies. The sensitivity studies performed here were restricted to a relatively small subset of the potential trajectory space for an entry vehicle. Additional studies would be required to ensuring that the altitude, Mach number, and angle-of-attack limits identified also apply at higher nominal angles of attack and at lower/higher altitudes as the sensitivity behavior may not be linear over the flight space.

The work presented suggests the inviscid/boundary-layer approach to aerothermal database generation is feasible. However, this investigation represents only a first step. It remains to be determined whether there is a practical way to implement this approach for application in the conceptual design environment where the vehicle configurations and missions may evolve rapidly. Ideally, an automated procedure could be formulated to drive the flowfield mesh creation and validation with the guidelines described; for example, checking the residual convergence and determining cell-size ratios for both the surface and flowfield meshes. However, at this stage of development, the engineer's perspective and judgment remain as the key to ensure that each solution is physically realistic and accurate. This study has clearly demonstrated that the generation of high-quality inviscid solutions required will be the greatest challenge to using the inviscid/boundary-layer approach in an automated, hands-off design system. In addition, suitable computational resources will be necessary to enable simulation of aerothermodynamic environments required for TPS design within the quick turn-around environment, typical for conceptual design studies. The initial findings of this study suggest that the implementation of the mid-fidelity aeroheating tool in an MDAO environment is feasible for generation of preliminary heating results, suitable for conceptual design. Thus, continued development towards the goal of implementation is recommended.

\section{Acknowledgments}

The authors would like to thank Jim Weilmuenster for his invaluable help and for sharing his wealth of knowledge, and Mike Aftosmis and Marian Nemec for their CART3D support. Many thanks to Hilmi Alkawahimi for his help with IDEA and Thomas West for providing all LAURA solutions presented.

\section{References}

\footnotetext{
${ }^{1}$ Freeman Jr., D. C., Talay, T. A., and Austin, R. E., "Reusable Launch Vehicle Technology Program," IAF Paper 96 V4.01, Oct. 1996.

${ }^{2}$ Gnoffo, P. A., Weilmuenster, K. J., Hamilton, II, H. H., Olynick, D. R., and Venkatapathy, E., "Computational Aerothermodynamics Design Issues for Hypersonic Vehicles," Journal of Spacecraft and Rockets, Vol. 36, No. 1, Jan-Feb 1999 , pp. 21-43.

${ }^{3}$ Wurster, K. E., Riley, C. J., and Zoby, E. V., "Engineering Aerothermal Analysis for X-34 Thermal Protection System Design," Journal of Spacecraft and Rockets, Vol. 36, No. 2, Mar-Apr 1999, pp. 216-228.

${ }^{4}$ Wurster, K. E. and Stone, H. W., "Aerodynamic Heating Environment Definiion/Thermal Protection System Selection for the HL-20," Journal of Spacecraft and Rockets, Vol. 30, No. 5, Sept - Oct 1993, pp. 549-557.

${ }^{5}$ Zoby, E. V., Thompson, R. A., and Wurster, K. E., "Aeroheating Design Issues for Reusable Launch Vehicles - A Perspective," AIAA 2004-2535, June 2004.

${ }^{6}$ Wurster, K. E., "Lifting Entry Vehicle Mass Reduction THrough Integrated Thermostructural/Trajectory Design," Journal of Spacecraft and Rockets, Vol. 20, No. 6, Nov - Dec 1983, pp. 589-596.

${ }^{7}$ Robinson, J. S., "An Overview of NASA's Integrated Design and Engineering Analysis (IDEA) Environment," AIAA 2011-2392, April 2011.

${ }^{8}$ Cunnington, R., Takahashi, T. T., and Bays, L. V., "Incorporation of Aerothermodynamic Analysis Tools into the Multi-Disciplinary Conceptual Design Process," AIAA 2007-406, Jan. 2007.
} 
${ }^{9}$ Engel, C. D. and Praharaj, S. C., "MINIVER Upgrade for the AVID System, Vol. I: LANMIN User's Manual," NASA CR-172212, 1983.

${ }^{10}$ Zoby, E. V. and Simmons, A. L., "Engineering Flowfield Method with Angle-of-Attack Applications," Journal of Spacecraft and Rockets, Vol. 22, July - August 1985, pp. 398-404.

${ }^{11}$ Wurster, K. E., Zoby, E. V., and Thompson, R. A., "Flowfield and Vehicle Parameter Influence on Results of Engineering Aerothermal Methods," Journal of Spacecraft and Rockets, Vol. 28, No. 1, Jan - Feb 1991, pp. 16-22.

${ }^{12}$ Fay, J. A. and Riddell, F. R., "Theory of Stagnation Point Heat Transfer in Dissociated Air," Journal of the Aeronautical Sciences, Vol. 25, No. 2, 1958, pp. 73-85.

${ }^{13}$ Sutton, K. and Graves Jr., R. A., "A General Stagnation Point Convective Heating Equation for Arbitrary Gas Mixtures," NASA TR R-376, Nov 1971.

${ }^{14}$ Zoby, E. V., "Approximate Relations for Laminar Heat-Transfer And Shear-Stress Functions in Equilibrium Dissociated Air," NASA TN D-4484, 1968.

${ }^{15}$ Zoby, E. V., Moss, J. N., and Sutton, K., "Approximate Convective-Heating Equations for Hypersonic Flows," Journal of Spacecraft and Rockets, Vol. 18, No. 1, 1979, pp. 64-70.

${ }^{16}$ Cooke, J. C., "An Axially Symmetric Analogue for General Three-Dimensional Boundary Layers," Ministry of Aviation: Aeronautical Research Council Reports and Memoranda, June 1959.

${ }^{17}$ Hamilton, H. H., DeJarnette, F. R., and Weilmuenster, K. J., "Application of Axisymmetric Analog for Calculating Heating in Three-Dimensional Flows," Journal of Spacecraft and Rockets, Vol. 24, No. 4, Jul - Aug 1987, pp. $296-302$.

${ }^{18}$ Hamilton, H. H., Greene, F. A., and DeJarnette, F. R., "Approximate Method for Calculating Heating Rates on ThreeDimensional Vehicles," Journal of Spacecraft and Rockets, Vol. 31, No. 3, May - June 1994, pp. 345-354.

${ }^{19}$ Riley, C. J., Kleb, W. L., and Alter, S. J., "Aeroheating Predictions for X-34 Using an Inviscid Boundary-Layer Method," Journal of Spacecraft and Rockets, Vol. 36, No. 2, Mar- Apr 1999, pp. 206-215.

${ }^{20}$ Hamilton, H. H., Weilmuenster, K. J., and DeJarnette, F. R., "Approximate Method for Computing Convective Heating on Hypersonic Vehicles Using Unstructured Grids," Journal of Spacecraft and Rockets, Vol. 51, No. 4, 2014, pp. 1288-1305.

${ }^{21}$ Mazaheri, A. R. and Kleb, B., "Exploring Hypersonic, Unstructured-Grid Issues through Structured Grids," AIAA 2007-4462, 2007.

${ }^{22}$ Gnoffo, P. A. and White, J. A., "Computational Aerothermodynamic Simulation Issues on Unstructured Grids," AIAA 2004-2371, 2004

${ }^{23}$ Candler, G. V., Barnhardt, M. D., Drayna, R. W., Nompelis, I., Peterson, D. M., and Subbareddy, P., "Unstructured Grid Approaches for Accurate Aeroheating Simulations," AIAA 2007-3959, 2007.

${ }^{24}$ Nemec, M., Aftosmis, M. J., and Wintzer, M., "Adjoint-Based Adaptive Mesh Refinement for Complex Geometries," AIAA 2008-0725, Jan. 2008.

${ }^{25}$ Peiro, J., P. J. and Morgan, K., "FELISA System Reference Manual and Users Guide," NASA CP-3291, May 1995.

${ }^{26}$ Bibb, K. L., Peraire, J., and Riley, C. J., "Hypersonic Flow Computations on Unstructured Meshes," AIAA Paper 1997-0625, January 1997.

${ }^{27}$ Gnoffo, P. A., Wood, W. A., Kleb, B., Alter, S., Glass, C., Padilla, J., Hammond, D., and White, J., "Functional Equivalence Acceptance Testing of FUN3D for Entry, Descent, and Landing Applications," AIAA 2013-2558, June 2013.

${ }^{28}$ Mazaheri, A., Gnoffo, P. A., Johnston, C. O., and Kleb, B., "LAURA Users Manual: 5.1-41601," NASA TM $2009,2009$.

${ }^{29}$ Gnoffo, P. A., Gupta, R. N., and Shinn, J. L., "Conservation Equations and Physical Models for Hypersonic Air Flows in Thermal and Chemical Nonequilibrium," NASA TP 2867, February 1989.

${ }^{30}$ Tartabini, P. V., Lepsch, R. A., Korte, J. J., and Wurster, K. E., "A Multidisciplinary Performance Analysis of a Lifting-Body Single-Stage-to-Orbit Vehicle," AIAA 2000-1045, 2000.

${ }^{31}$ Wurster, K. E., "An Assessment of the Impact of Transition on Advanced Winged Entry Vehicle Thermal Protection System Mass," AIAA-81-1090, 1981.

${ }^{32}$ Anderson Jr., J. D., Hypersonic and High-Temperature Gas Dynamics, American Institute of Aeronautics and Astronautics, Reston, VA, 2nd ed., 2006.

${ }^{33}$ Anon, "Adaptive Modeling Language, Version 5.0B5, Reference Manual," TechnoSoft, Inc., 2010.

${ }^{34}$ Pulsonetti, M. V. and Thompson, R. A., "LAURA Aerothermodynamic Computations for Space Shuttle Columbia STS107 Baseline and Damage Scenarios," AIAA 2001-2278, 2001.

${ }^{35}$ Thompson, R. A., Lessard, V., Jentink, T., and Zoby, E. V., "Analysis of Compression Pad Cavities for the Orion Heatshield," AIAA 2009-1575, 2009.

${ }^{36}$ Edquist, K. T., Hollis, B. R., Johnston, C. O., Bose, D., White, T. R., and Mahzari, M., "Mars Science Laboratory Heat Shield Aerothermodynamics: Design and Reconstruction," Journal of Spacecraft and Rockets, Vol. 51, No. 4, July-August 2014, pp. 1106-1124.

${ }^{37}$ Cleary, J. W., "Effects of Angle of Attack and Bluntness on Laminar Heating-Rate Distributions of a 15deg Cone at a Mach Number of 10.6," NASA TN D-5450, Oct. 1969.

${ }^{38}$ Throckmorton, D. A. and Hartung, L. C., "Space Shuttle Orbiter Entry Lee-Side Heat-Transfer Data—STS -28," NASA RP-1306, September 1993.

39 "U.S. Standard Atmosphere, National Oceanic and Atmospheric Administration, NASA, and U.S. Air Force," Washington, D.C., 1976. 